\title{
Playing with ultrasound in complex media
}

\author{
Arnaud Tourin
}

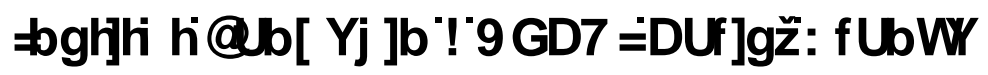




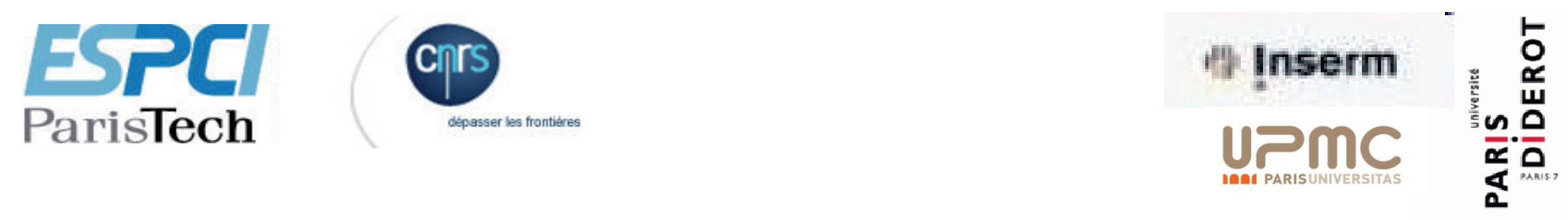

\section{Playing with ultrasound in complex media}

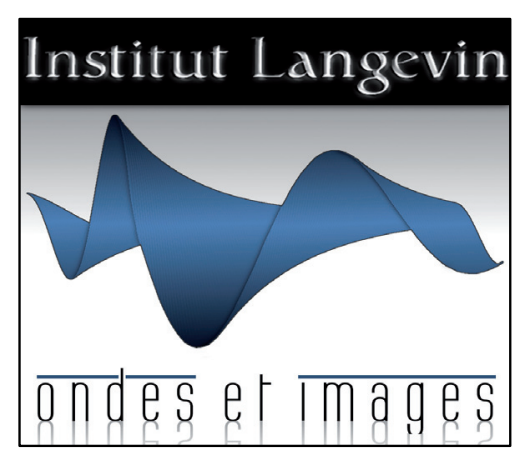

Arnaud Tourin

Cargèse - July, 13, 2010 


\section{The Langevin Institute}

Founded in january 2009 to gather people from

$>$ LOA (M. FINK) : ultrasound, sound, seismic waves, microwaves

$>\operatorname{LOP}(\mathrm{C} . \mathrm{BOCCARA})$ : optics, ultrasound

New devices for manipulating waves in various kinds of media

$>$ Time Reversal Mirror

$>$ Multiwave Imaging

$>$ IR SNOM, thermal radiation STM

- Medical imaging \& therapy, non destructive testing, underwater acoustics, seismology, telecommunications, tactile objects,...

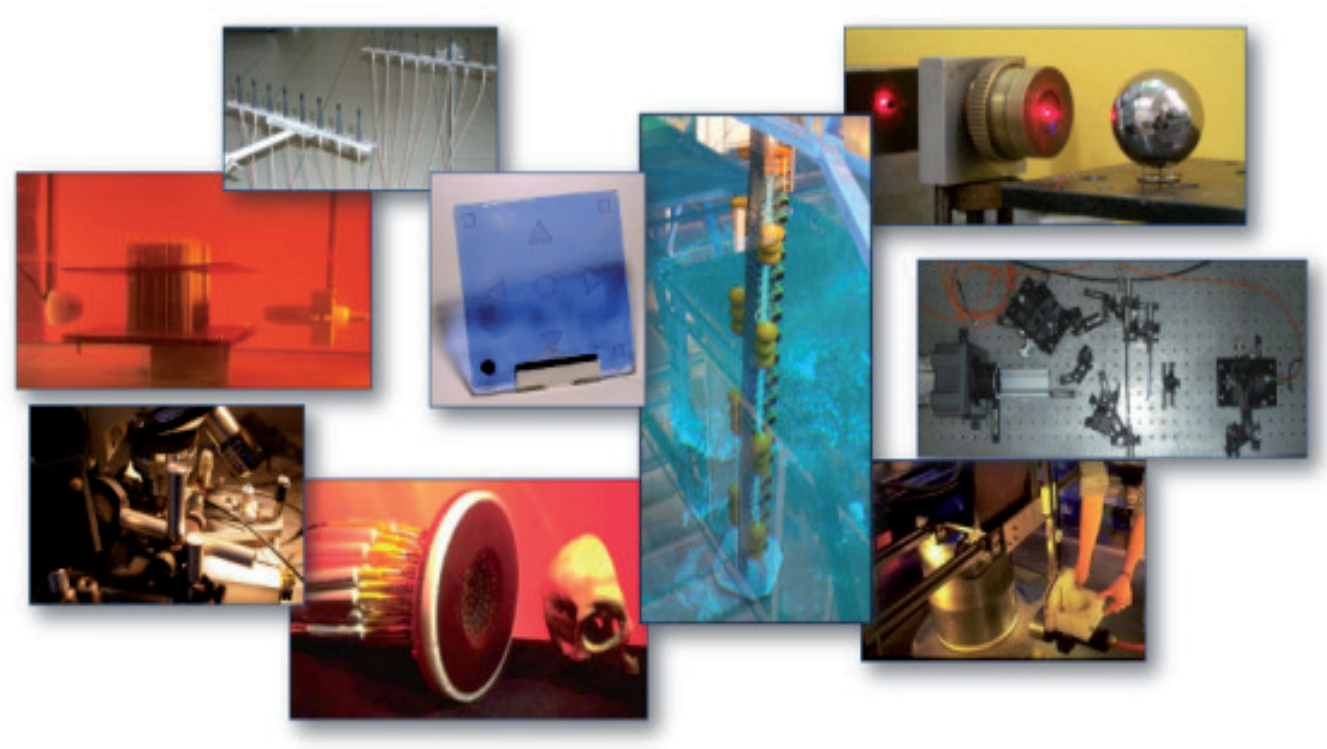

- Fundamental physics/applications/companies

$>$ Echosens, Sensitive Object, SSI, TRCOM, LLTech ( 200) 


\section{The Langevin Institute}

\section{2 people}

$>\quad 34$ permanent researchers

$>14$ for the technical \& administrative support

> 29 Phd students

$>15$ Post-Doc

$>$ One Director (M. Fink) and two vice directors (R. Carminati and AT)

5 groups

> Acoustic waves \& microwaves in complex media

$>$ Nanophotonics \& Optical waves in diffusive media

$>$ Wave Physics for medicine \& Biology

$>$ Detection, Imaging, and characterization 


\section{Acoustic waves \& microwaves in random media}

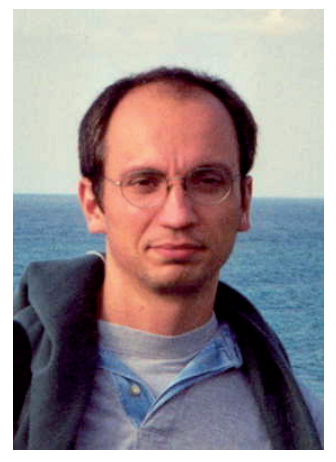

Julien de Rosny

(Research scientist)

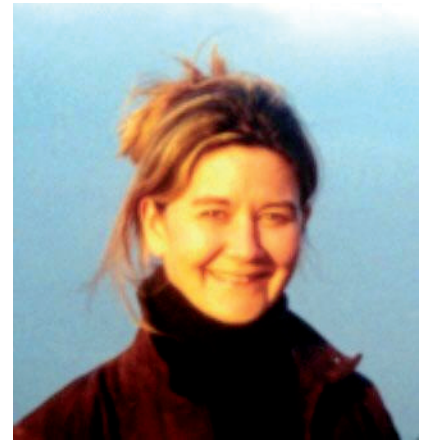

Agnès Maurel

(Research scientist)

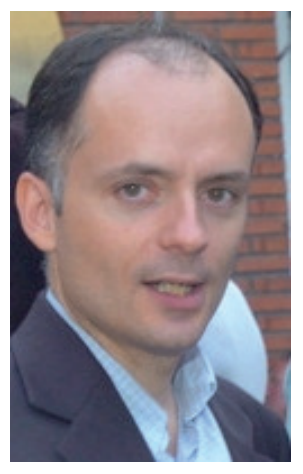

Arnaud Tourin

(Professor)

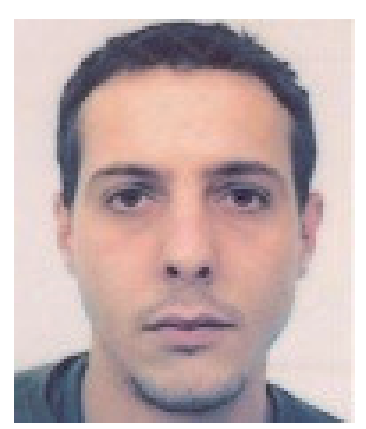

Geoffroy Lerosey (Research scientist)

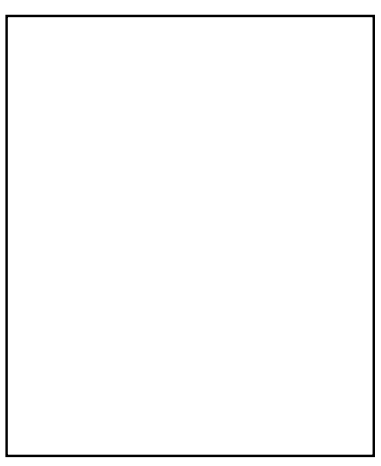

Arnaud Derode

(Professor)

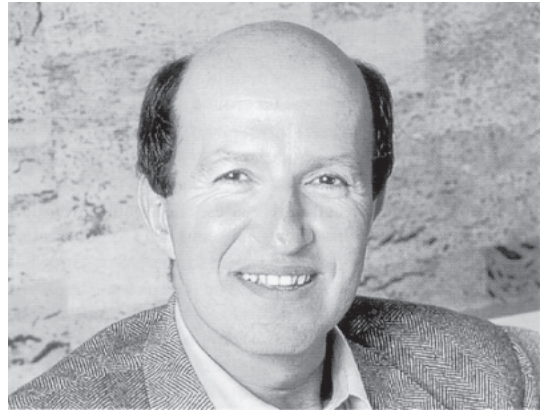

Mathias Fink

(Professor)

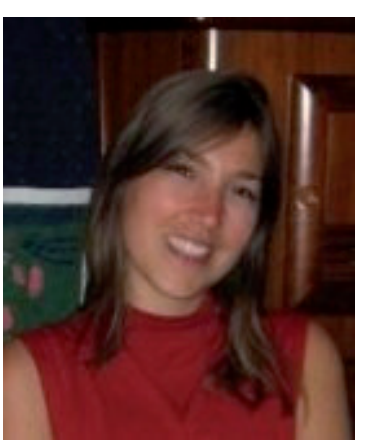

Marie Müller

(Associate professor)

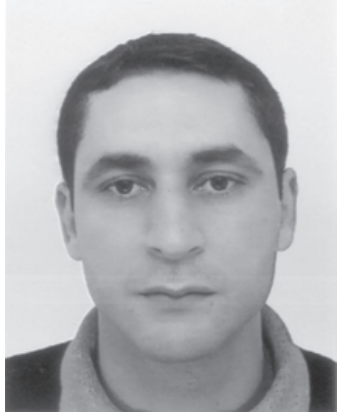

Abdel Ourir

(Engineer)

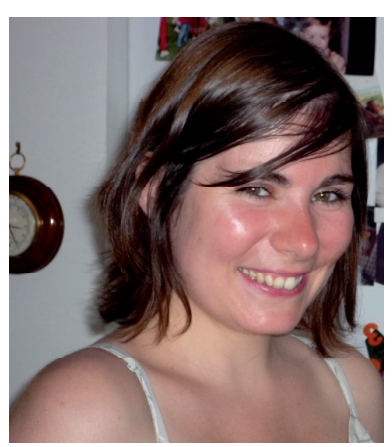

Alice Bretagne (PhD student) 


\section{OUTLINE}

$\checkmark$ Wave propagation in random media

The ballistic and coherent waves

The incoherent wave and the diffusion approximation

$\rightarrow$ Beyond the DA : the backscattering cone

- Using disorder for controlling wave propagation

Disorder for guiding ultrasound

- Disorder for focusing ultrasound

A random (or ordered) bubbly medium for filtering US 


\section{Mesoscopic physics with ultrasound}

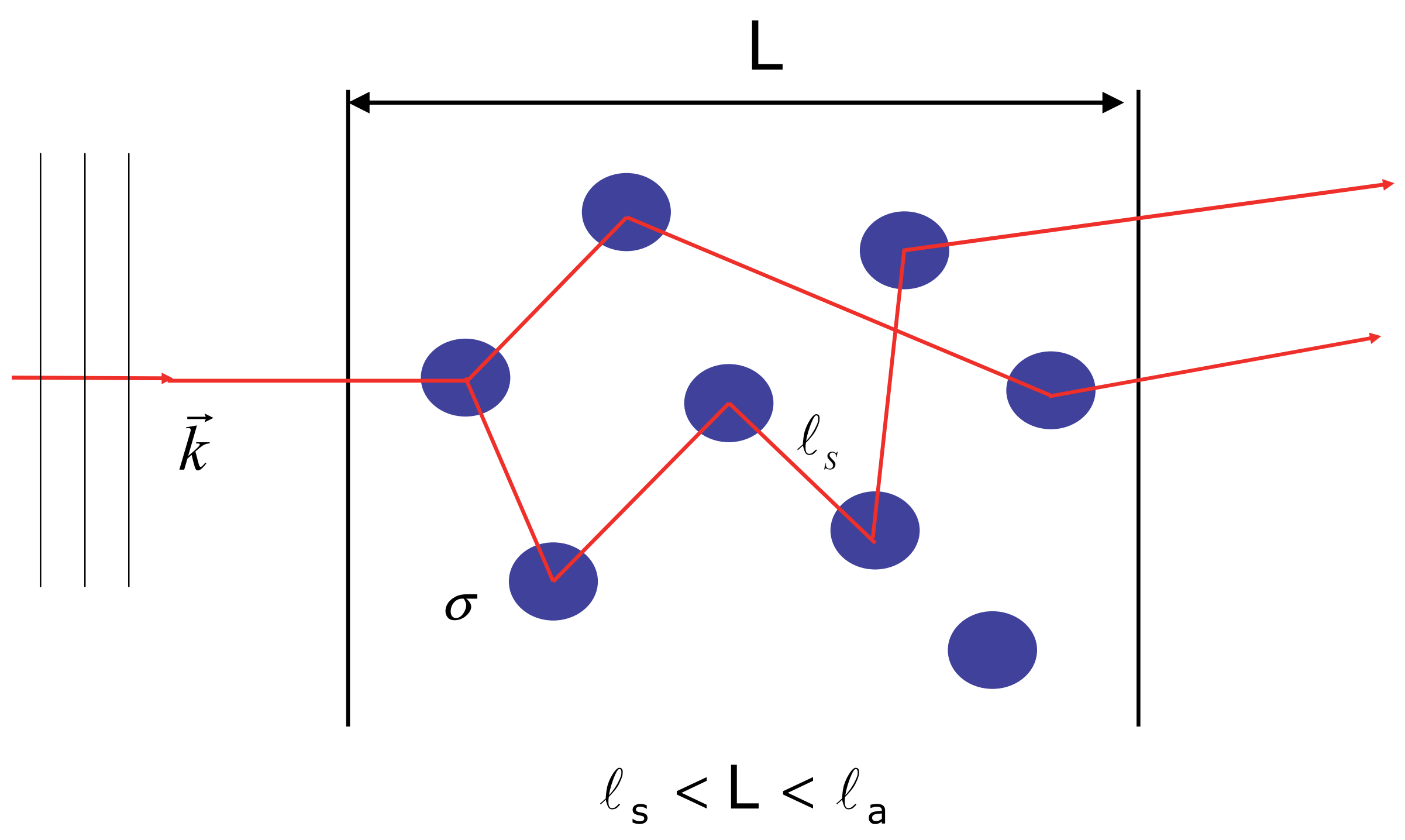




\section{Experimental approach : measuring the S matrix}
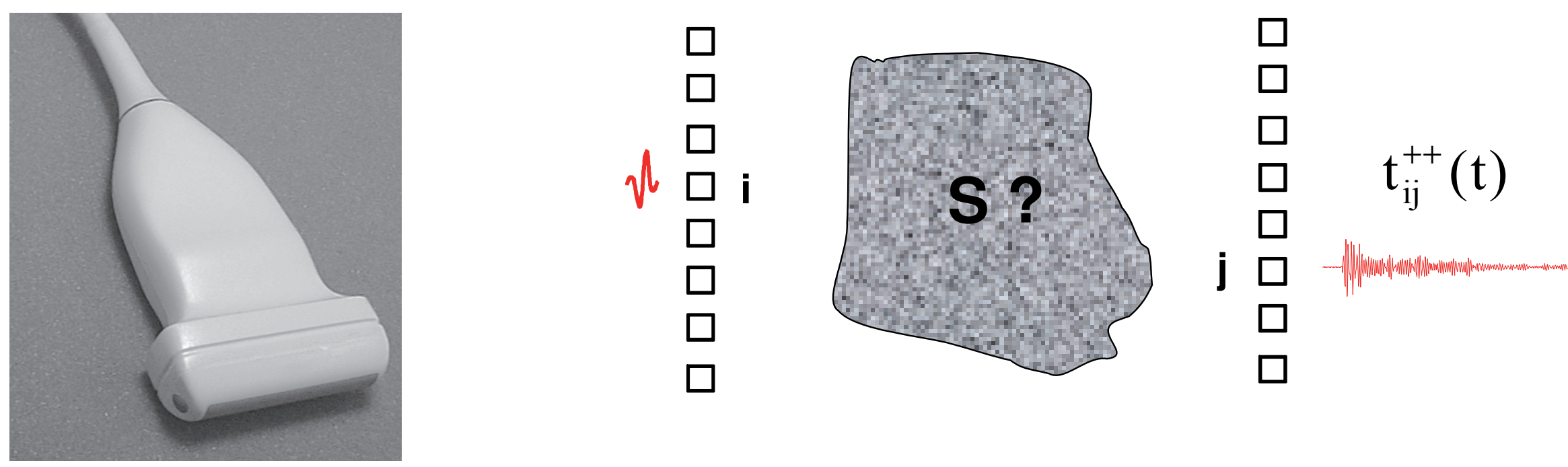

Transducer arrays

$\mathrm{f} \sim 500 \mathrm{kHz}$ à $5 \mathrm{MHz}$

$$
\mathrm{S}=\left(\begin{array}{ll}
\mathrm{r}^{-+} & \mathrm{t}^{--} \\
\mathrm{t}^{++} & \mathrm{r}^{+-}
\end{array}\right)
$$

$$
\lambda \sim \mathrm{mm}
$$




\section{Experimental approach : measuring the S matrix}

$\square$ (average) time-resolved transmitted amplitude / intensity

$\square$ (average) backscattered amplitude or intensity (BS Cone)

$\square$ Perform a SVD and study the statistics of the singular values

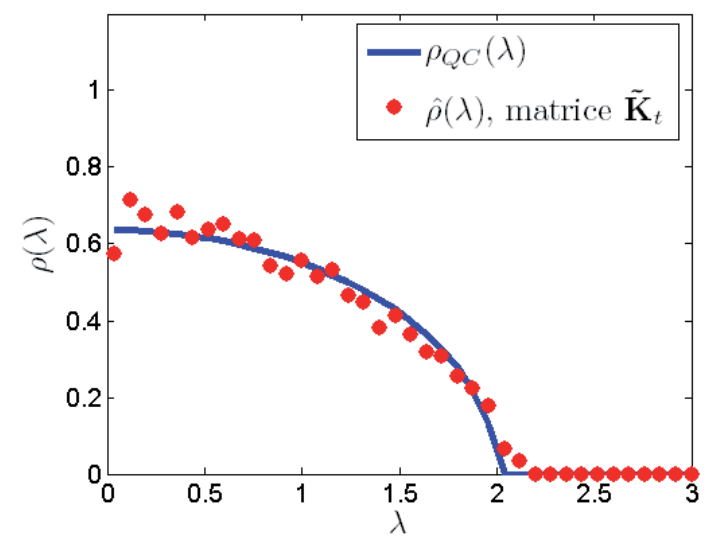

$\square$ Focusing using time reversal, wavefront shaping, inverse filter 


\section{Experimental approach :}

\section{what can be measured with ultrasound?}
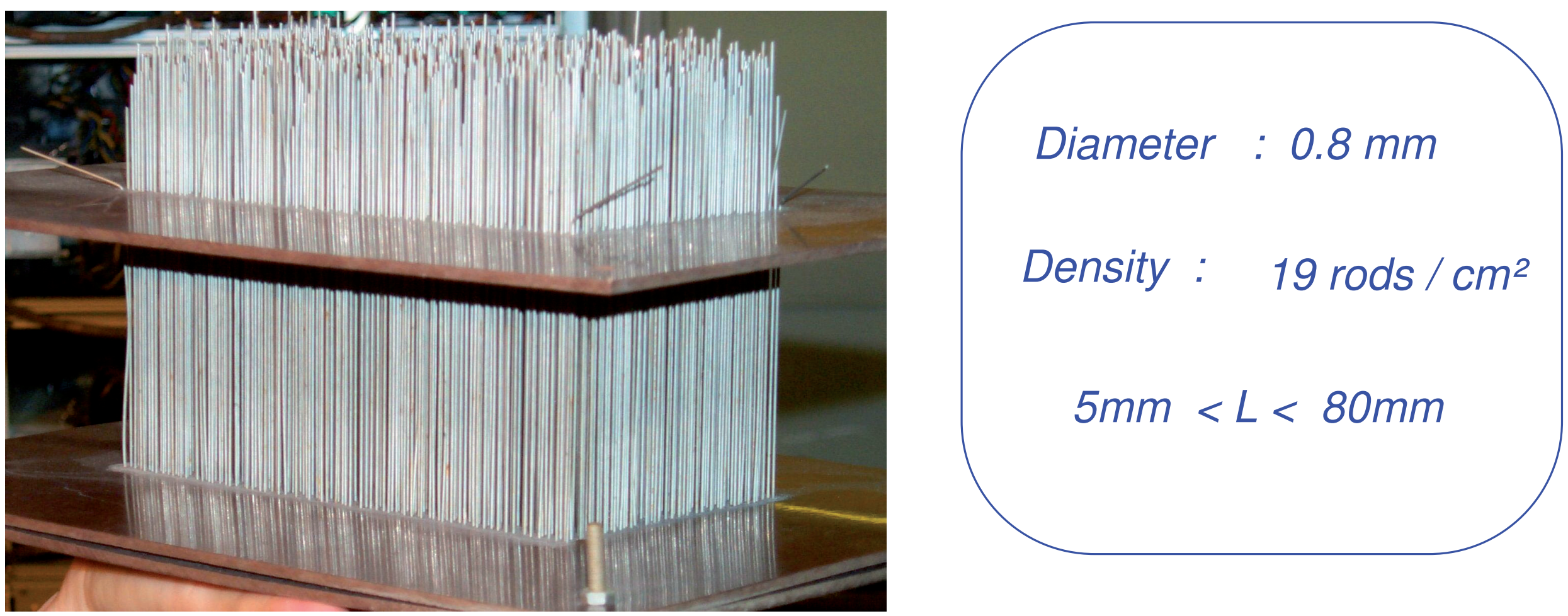


\section{Experimental approach}

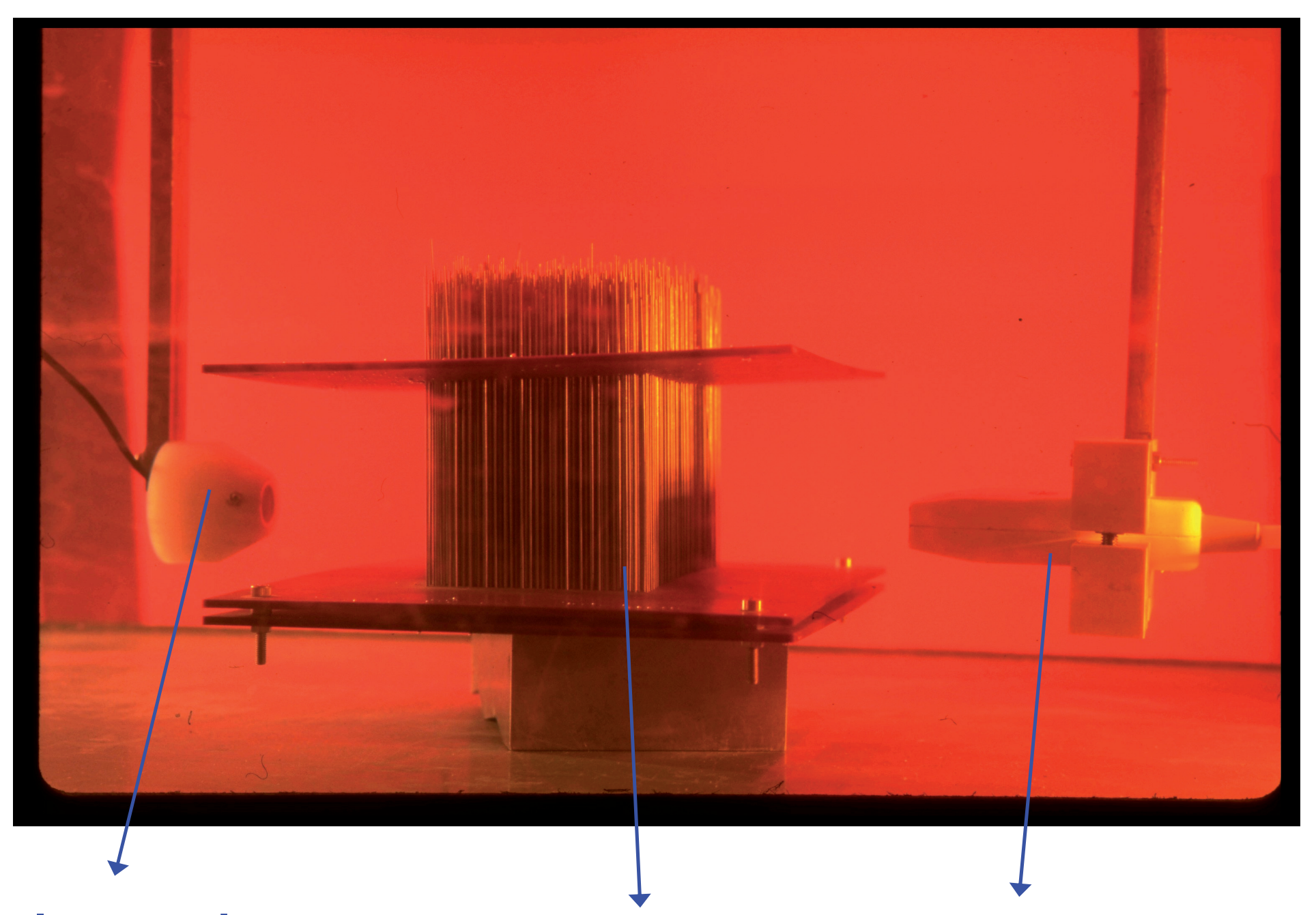

Single transducer $v=3.2 \mathrm{MHz}, \lambda=0.48 \mathrm{~mm}$
2D random sample 128-transducer array Pitch : $0.42 \mathrm{~mm}$ 


\section{Spatio-temporal distribution of the amplitude}
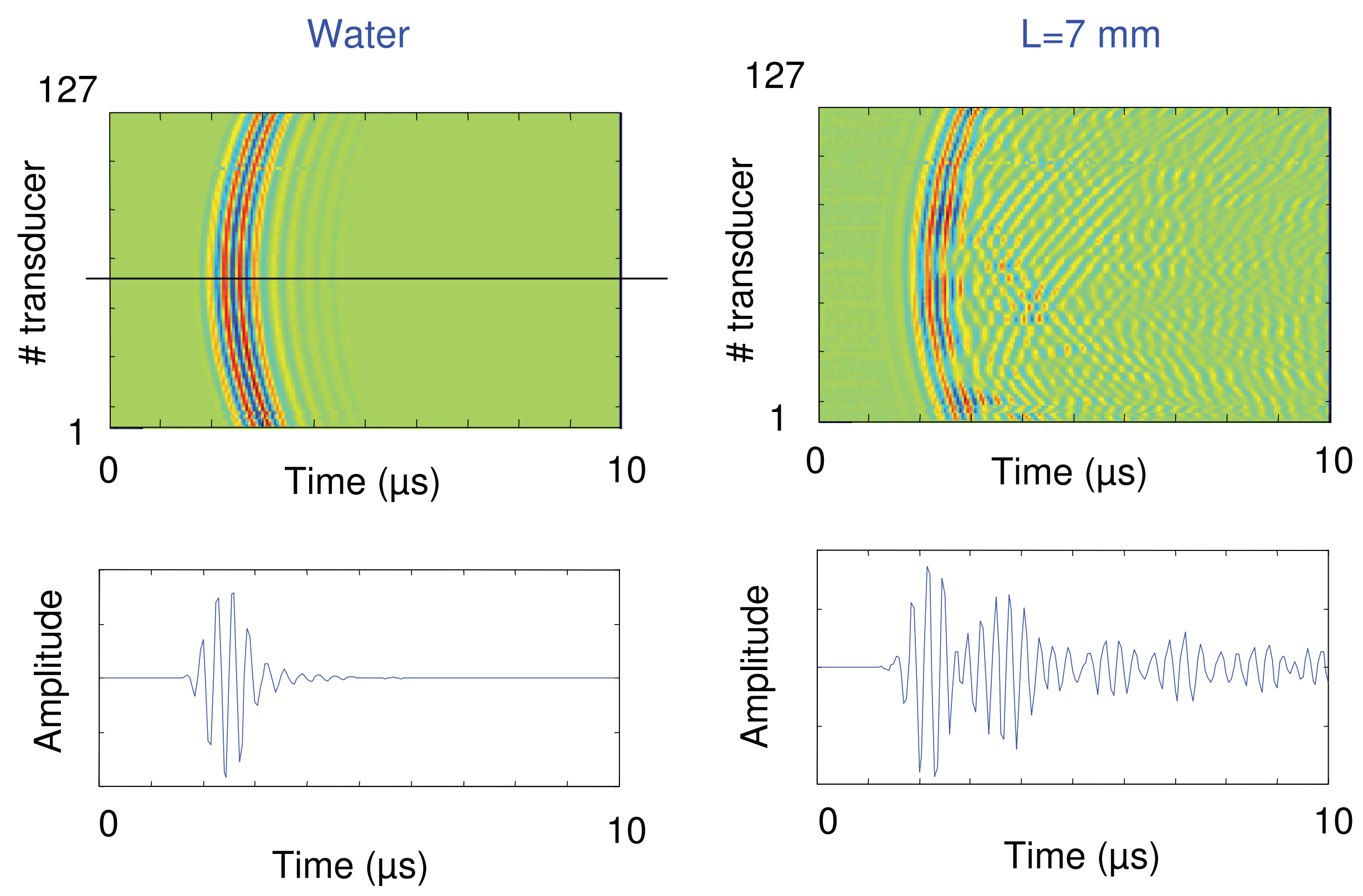


\section{Spatio-temporal distribution of the amplitude}
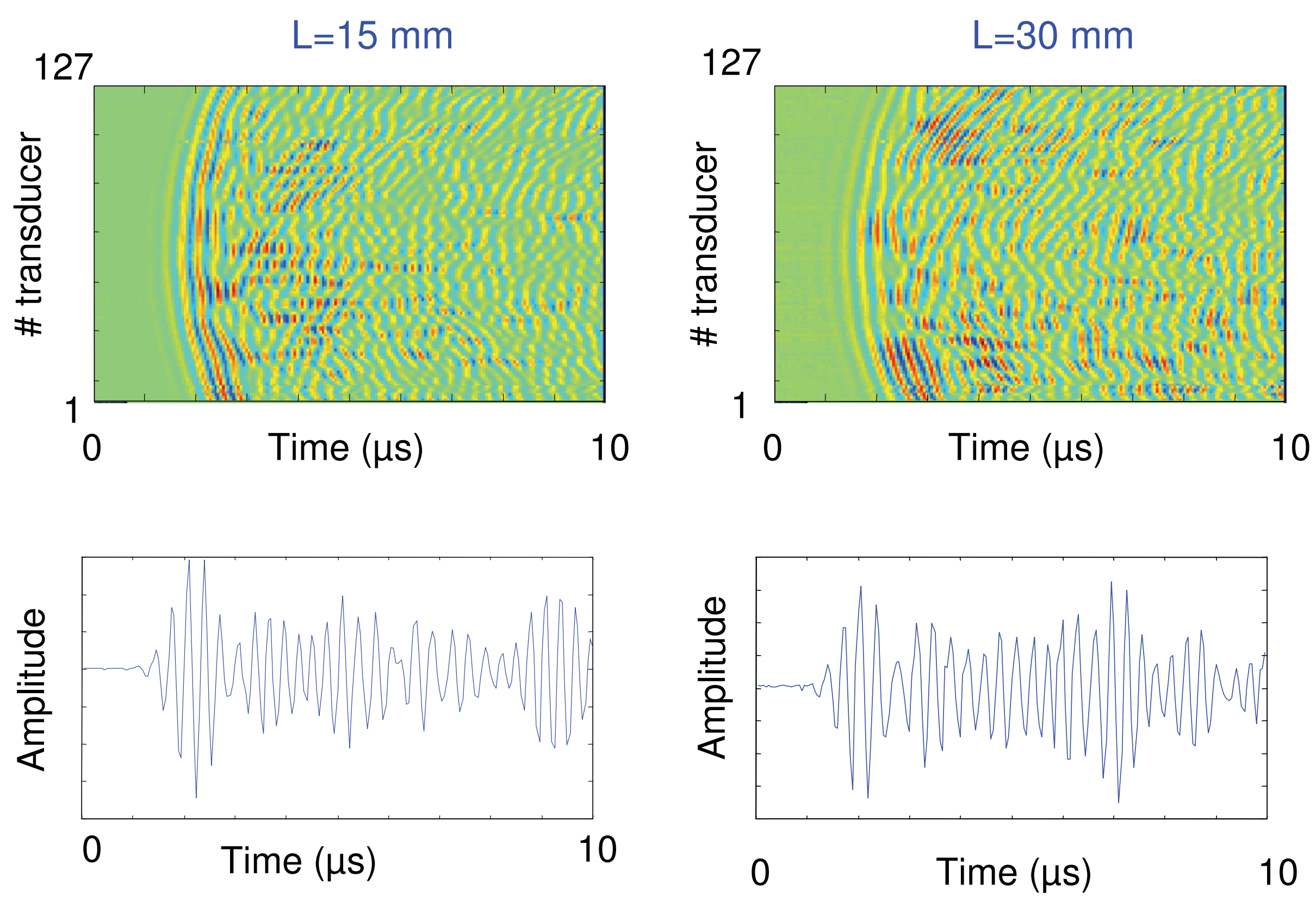


\section{Spatio-temporal distribution of the amplitude}

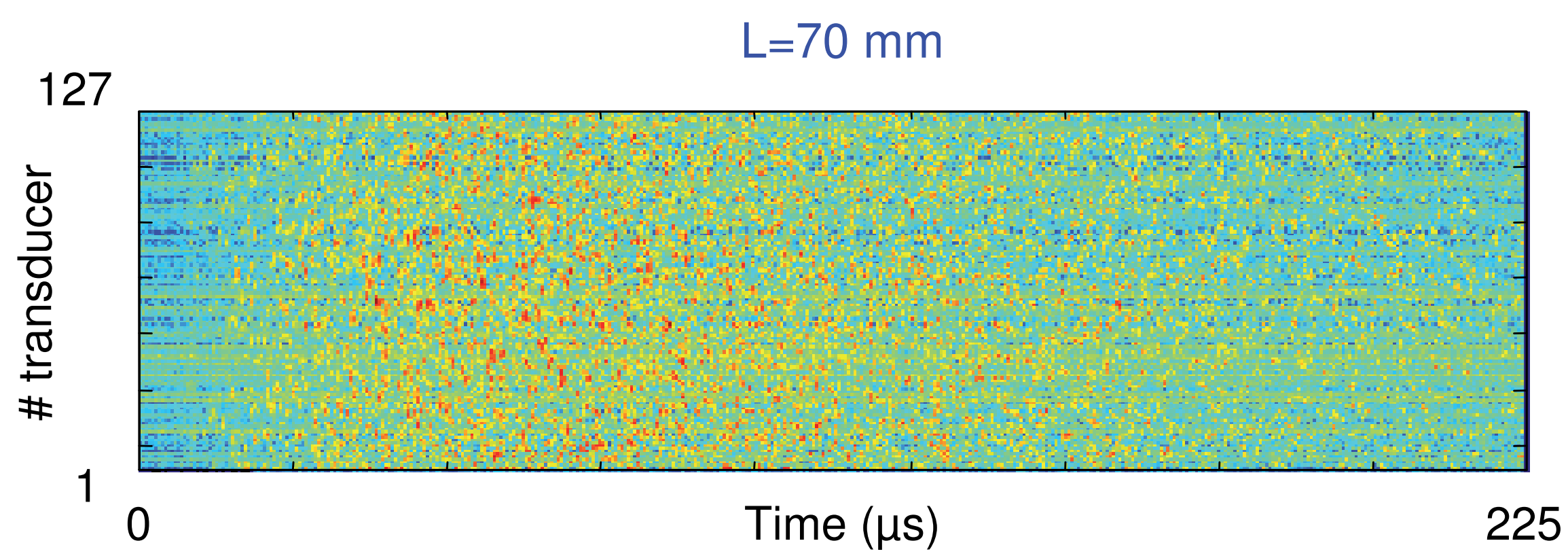

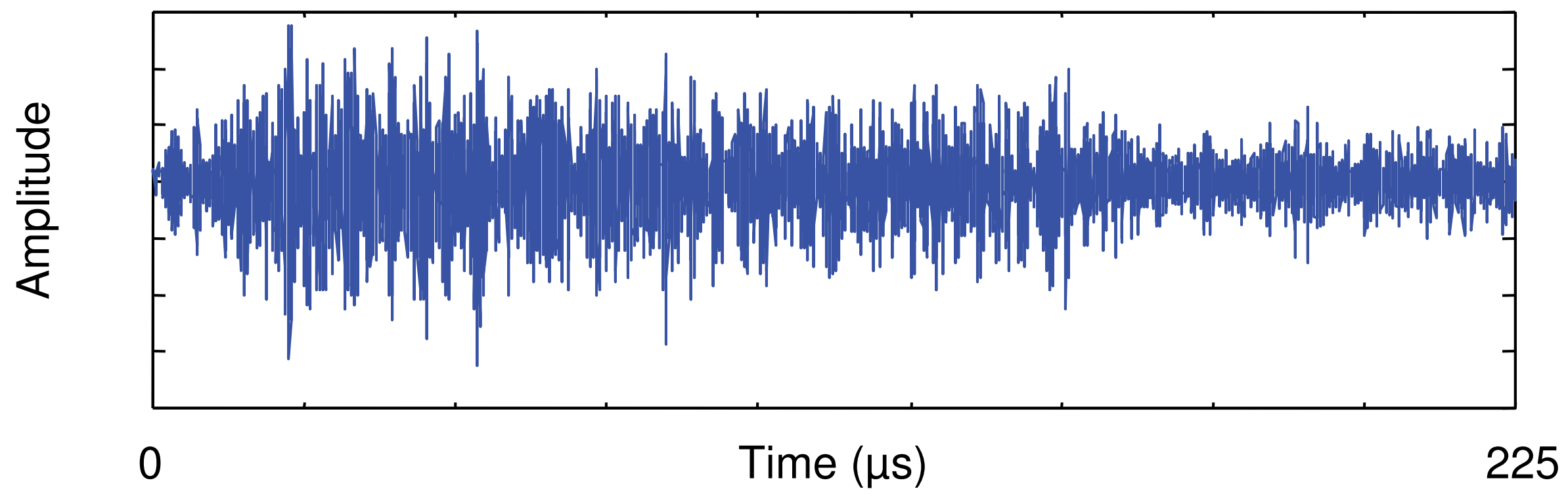




\section{The wave equation in a heterogeneous medium}

\section{Classical (acoustic) wave}

\section{Quantum wave}

$$
\begin{array}{c|c}
\Delta \Psi(\mathbf{r}, t)=\frac{1}{\mathrm{c}(\mathbf{r})^{2}} \frac{\partial^{2} \Psi(\mathbf{r}, t)}{\partial t^{2}} & \left(-\frac{\hbar^{2}}{2 \mathrm{~m}} \Delta+\mathrm{V}(\mathbf{r})\right) \Psi(\mathbf{r}, \mathrm{t})=\mathrm{i} \hbar \frac{\partial \Psi(\mathbf{r}, \mathrm{t})}{\partial} \\
k_{0}^{2}=\frac{\omega^{2}}{c_{0}^{2}} & k_{0}^{2}=\frac{2 m \omega}{\hbar^{2}} \\
\sigma(\mathbf{r})=k_{0}^{2}(\omega)-k^{2}(\omega) & \sigma(\mathbf{r})=\frac{2 m V(\mathbf{r})}{\hbar^{2}}
\end{array}
$$

$$
\Delta \Psi(\omega, \mathbf{r})+k_{0}^{2} \Psi(\omega, \mathbf{r})=\sigma(\mathbf{r}) \Psi(\omega, \mathbf{r})
$$

$$
\Psi(\omega, \mathbf{r})=\Psi_{0}(\omega, \mathbf{r})+\int_{V} G_{0}\left(\omega, \mathbf{r}-\mathbf{r}^{\prime}\right) \sigma\left(\mathbf{r}^{\prime}\right) \Psi\left(\mathbf{r}^{\prime}\right) d \mathbf{r}^{\prime}
$$




\section{The wave equation in a heterogeneous medium}

\section{The Born expansion}

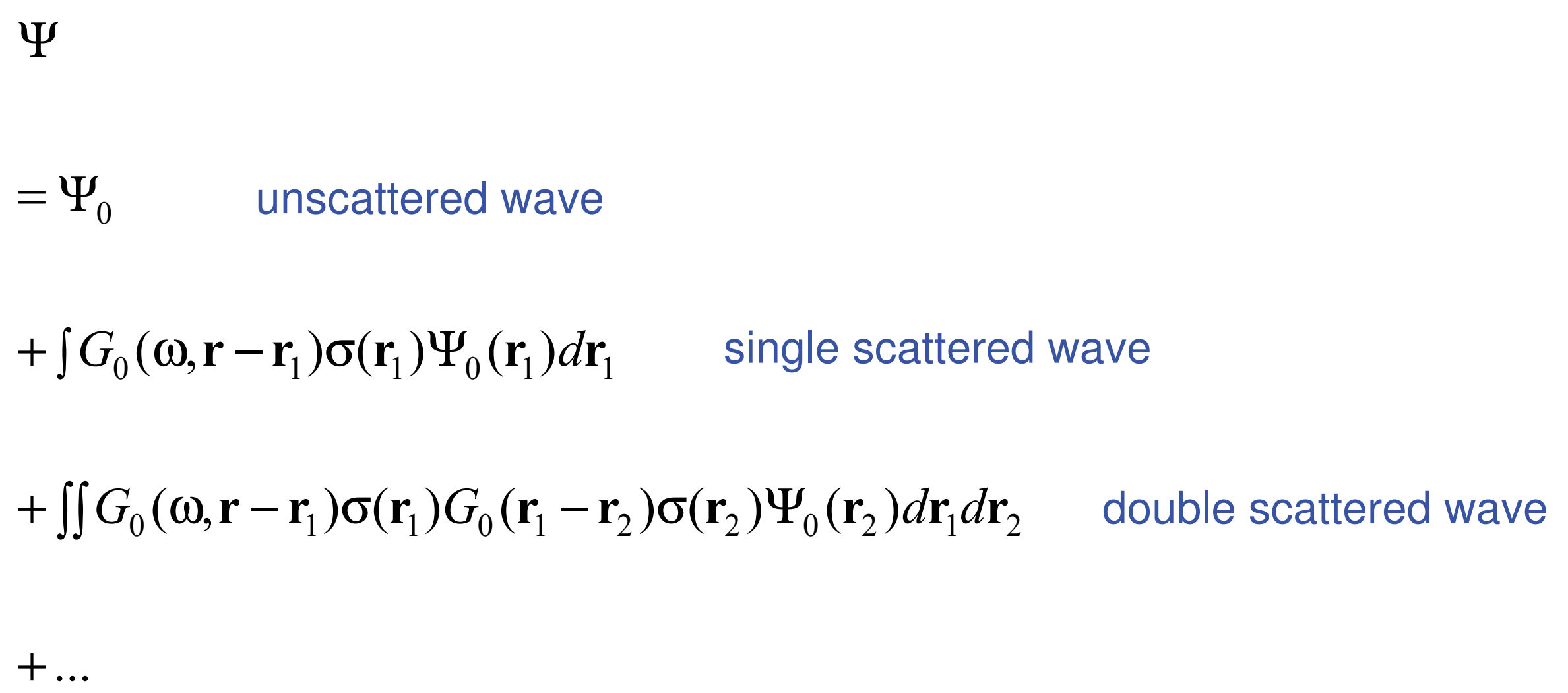




\section{The Dyson equation}

$$
\begin{aligned}
& \Delta \mathrm{G}\left(\omega, \mathbf{r}, \mathbf{r}^{\prime}\right)+\mathrm{k}_{0}^{2} \mathrm{G}\left(\omega, \mathbf{r}, \mathbf{r}^{\prime}\right)=\sigma(\mathbf{r}) \mathrm{G}\left(\omega, \mathbf{r}, \mathbf{r}^{\prime}\right)+\delta\left(\mathbf{r}-\mathbf{r}^{\prime}\right) \\
& \left\langle\mathrm{G}\left(\omega, \mathbf{r}, \mathbf{r}^{\prime}\right)\right\rangle=\mathrm{G}_{0}\left(\omega, \mathbf{r}-\mathbf{r}^{\prime}\right)+\int \mathrm{G}_{0}\left(\omega, \mathbf{r}-\mathbf{r}_{1}\right)\left\langle\sigma\left(\mathbf{r}_{1}\right) \mathrm{G}\left(\omega, \mathbf{r}_{1}, \mathbf{r}^{\prime}\right)\right) d \mathbf{r}_{1}
\end{aligned}
$$

$\left\langle G\left(\omega, \mathbf{r}-\mathbf{r}^{\prime}\right)\right\rangle=G_{0}\left(\omega, \mathbf{r}-\mathbf{r}^{\prime}\right)+\iint G_{0}\left(\omega, \mathbf{r}-\mathbf{r}_{1}\right) \Sigma\left(\omega, \mathbf{r}_{1}-\mathbf{r}_{2}\right)\left\langle G\left(\omega, \mathbf{r}_{2}-\mathbf{r}^{\prime}\right)\right\rangle d \mathbf{r}_{1} d \mathbf{r}_{2}$

$$
\mathrm{I}_{\text {coh }}=\left|\left\langle\mathrm{G}_{\omega}\left(\mathbf{r}, \mathbf{r}_{0}\right)\right\rangle\right|^{2} \propto \frac{\operatorname{expik} \mathrm{e}_{\mathrm{e}}\left|\mathbf{r}-\mathbf{r}_{0}\right|}{4 \pi\left|\mathbf{r}-\mathbf{r}_{0}\right|} \exp -\frac{\left|\mathbf{r}-\mathbf{r}_{0}\right|}{\ell_{\mathrm{S}}} \quad \text { Beer-Lambert }
$$

$$
l_{S}=1 / n \sigma \quad \text { Scattering mean free path }
$$




\section{How to build an estimator of the ensemble average?}

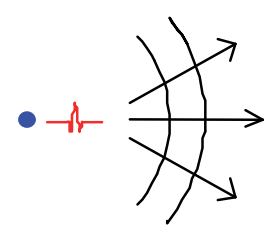

Source

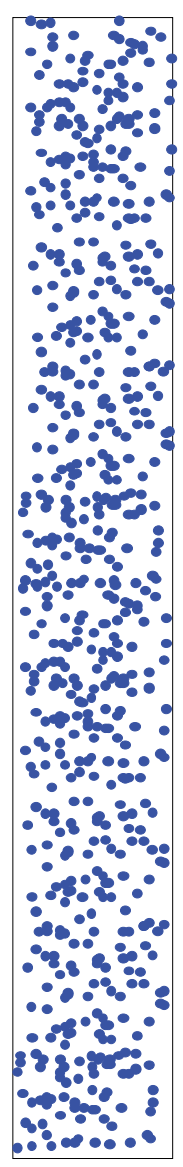

Receiving array 


\section{The coherent wave}

\section{$\mathrm{L}=30 \mathrm{~mm}$}
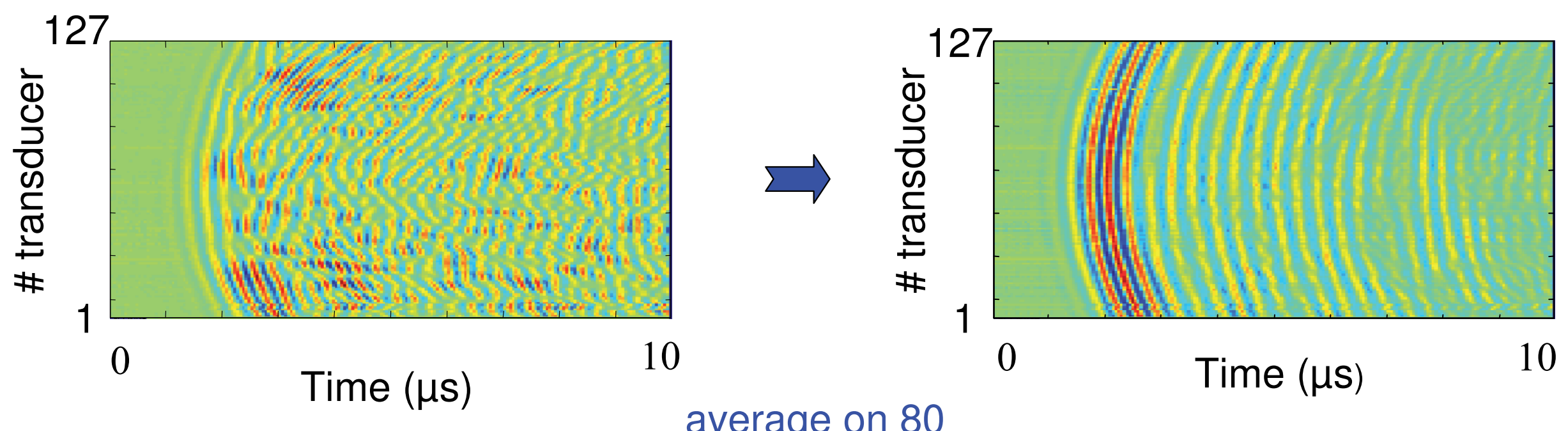

average on 80

Configurations
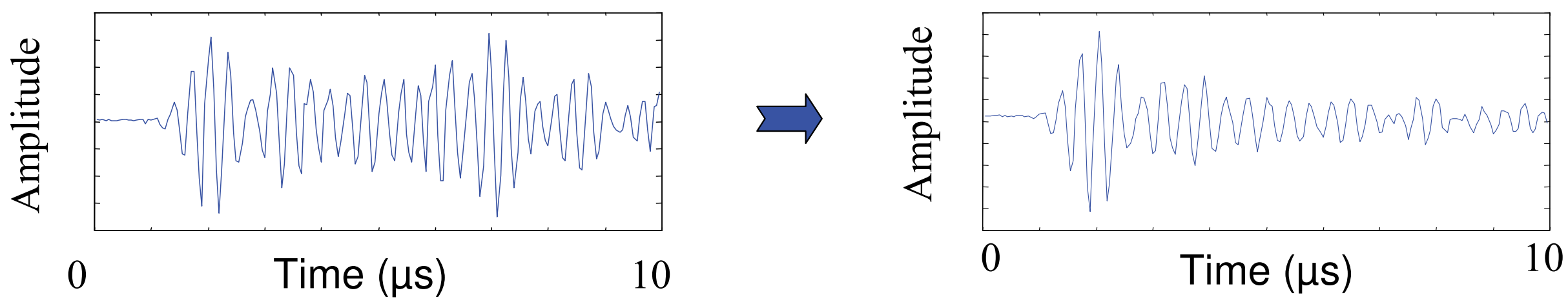


\section{The coherent wave}

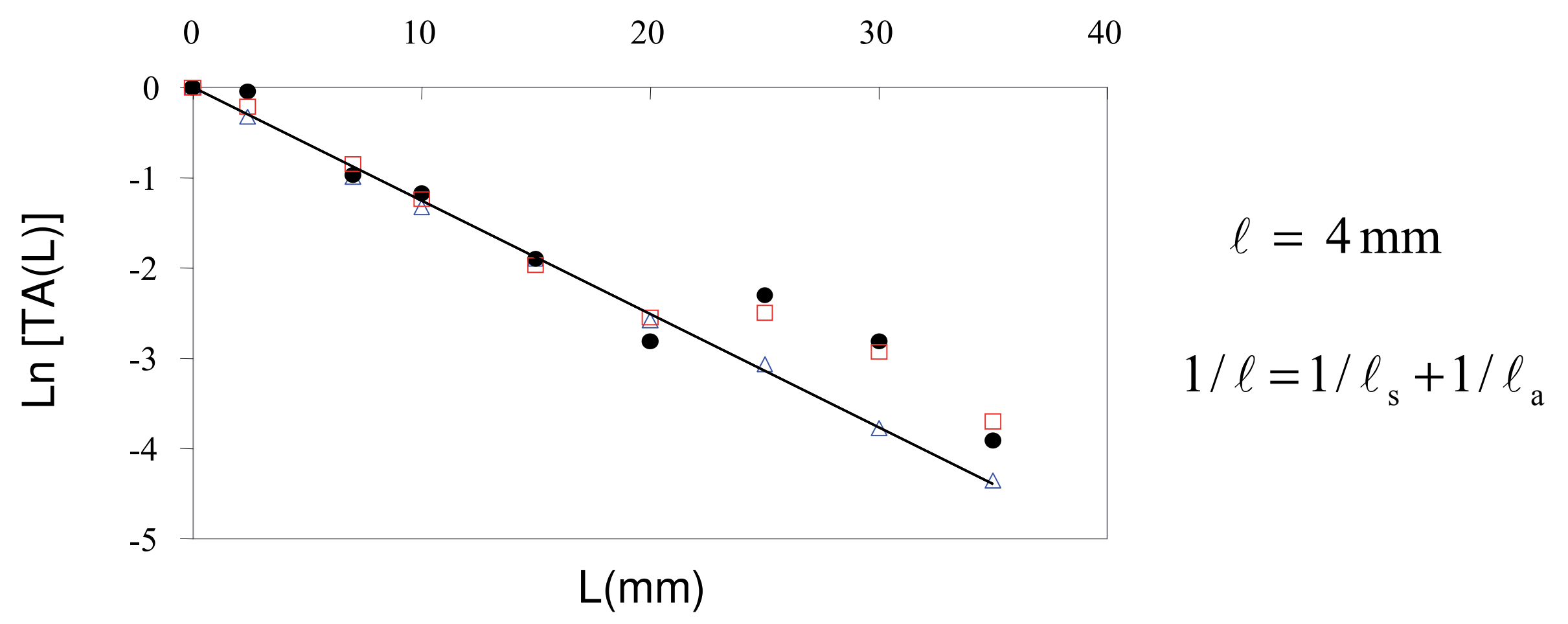

- Ballistic amplitude

$\square \quad$ Ballistic amplitude averaged over the 127 transducers

$\triangle$ Ballistic amplitude averaged over 80 random sample positions 


\section{The coherent wave}
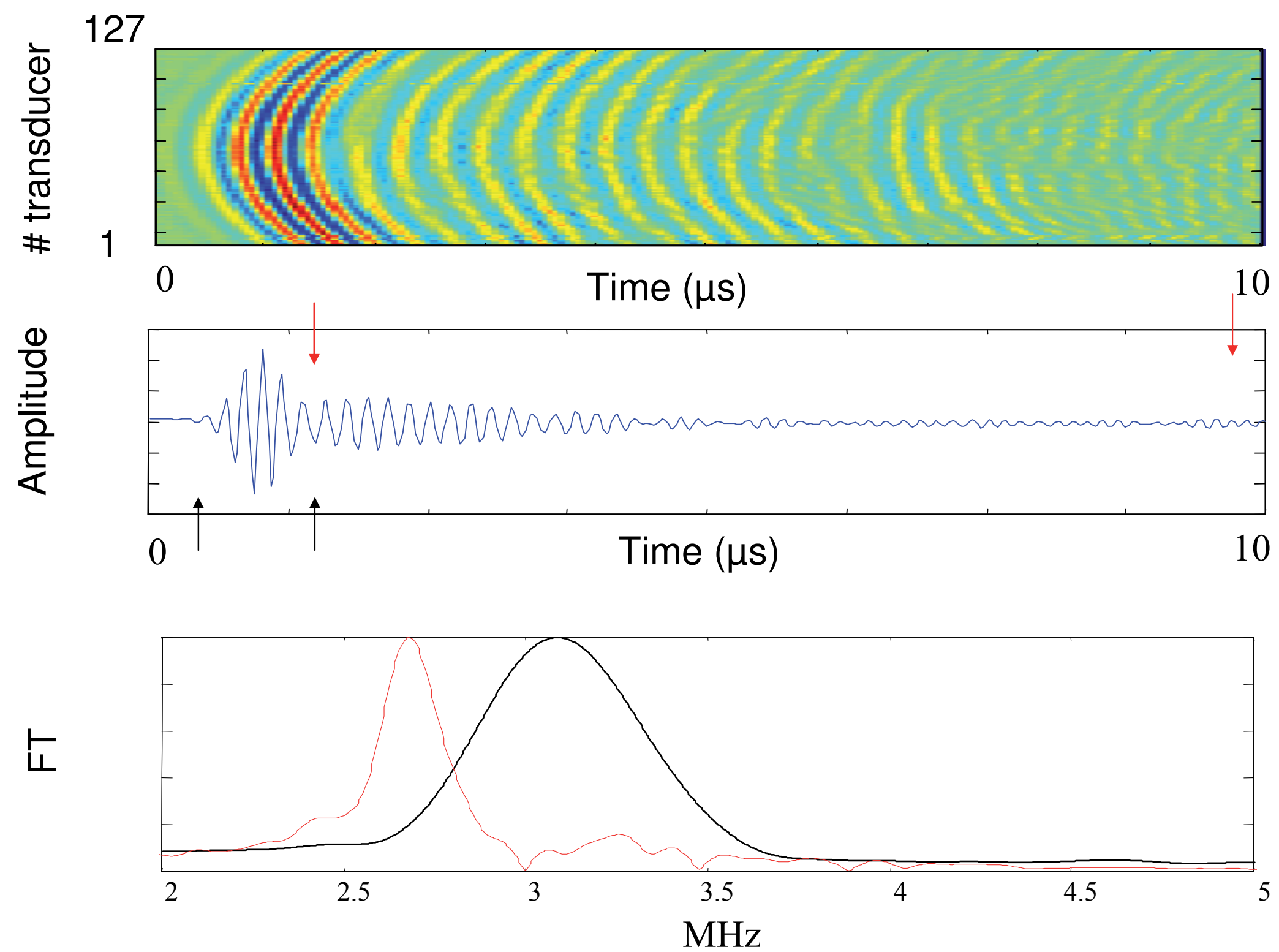


\section{The coherent wave}
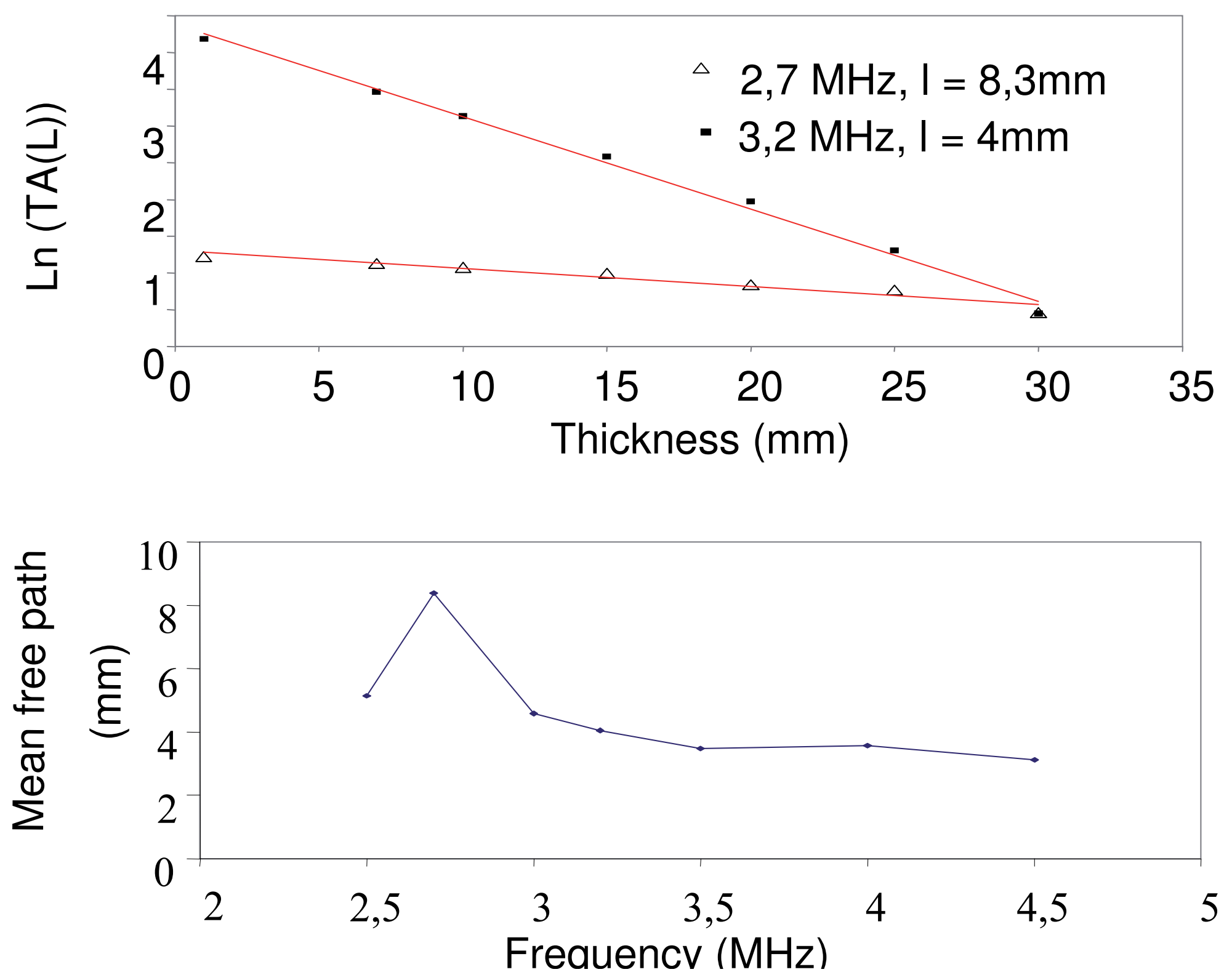


\section{Scattering from a single inclusion}

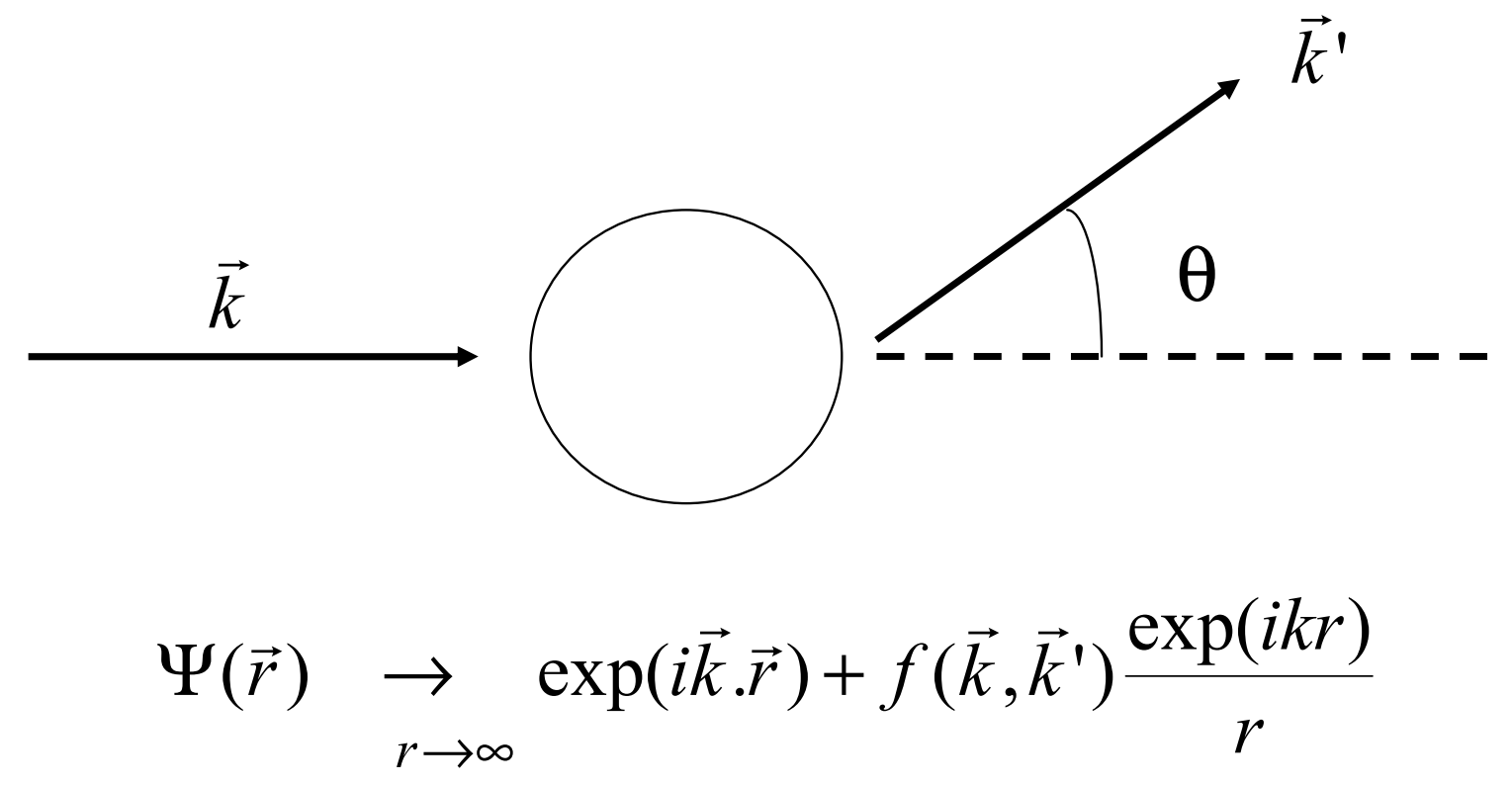

Scattering cross section

$$
\sigma=\frac{P_{S}}{d P_{i n c} / d S}=2 \pi \int_{0}^{\pi} \sin \theta|f(\theta, k)|^{2} d \theta
$$

Optical theorem

$$
\sigma=\frac{4 \pi}{k_{0}} \operatorname{Im} f\left(k_{0}, 0\right)
$$




\section{Scattering from a single inclusion}

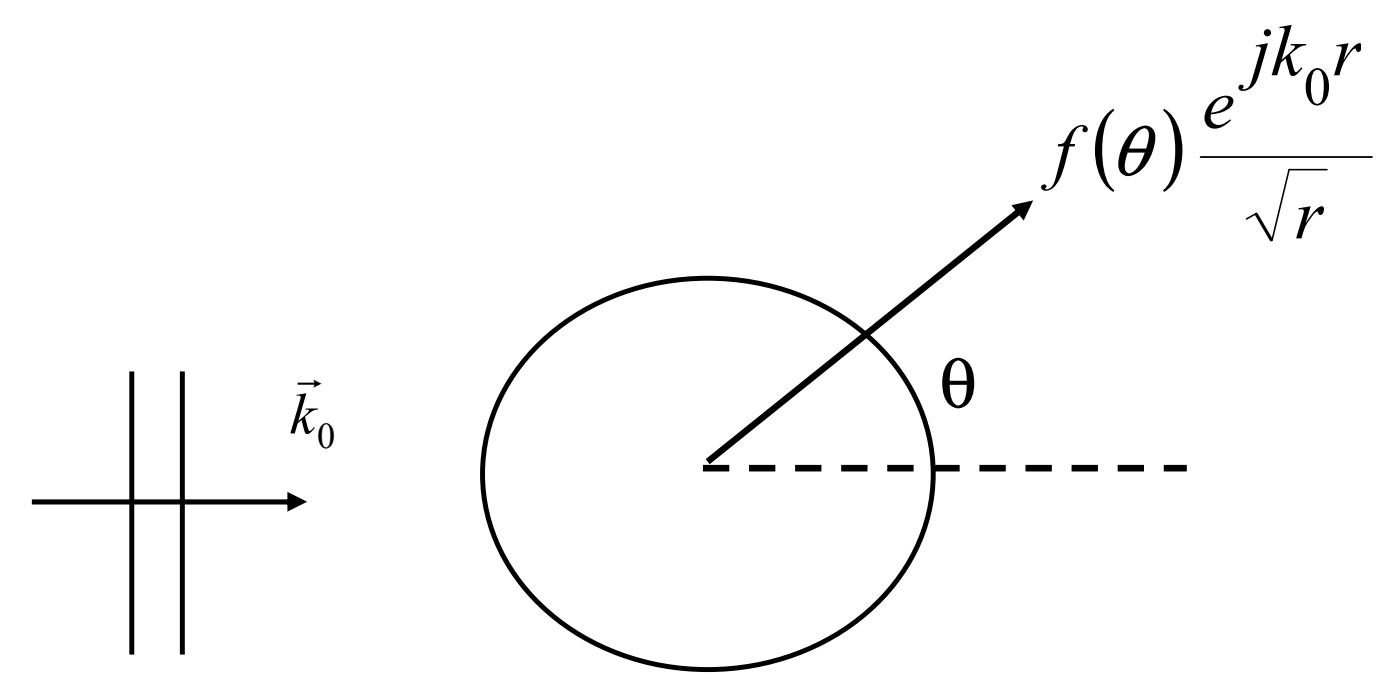

$$
\begin{gathered}
\sigma(\theta)=|f(\theta)|^{2} \\
\sigma=\int_{0}^{2 \pi} \sigma(\theta) d \theta
\end{gathered}
$$

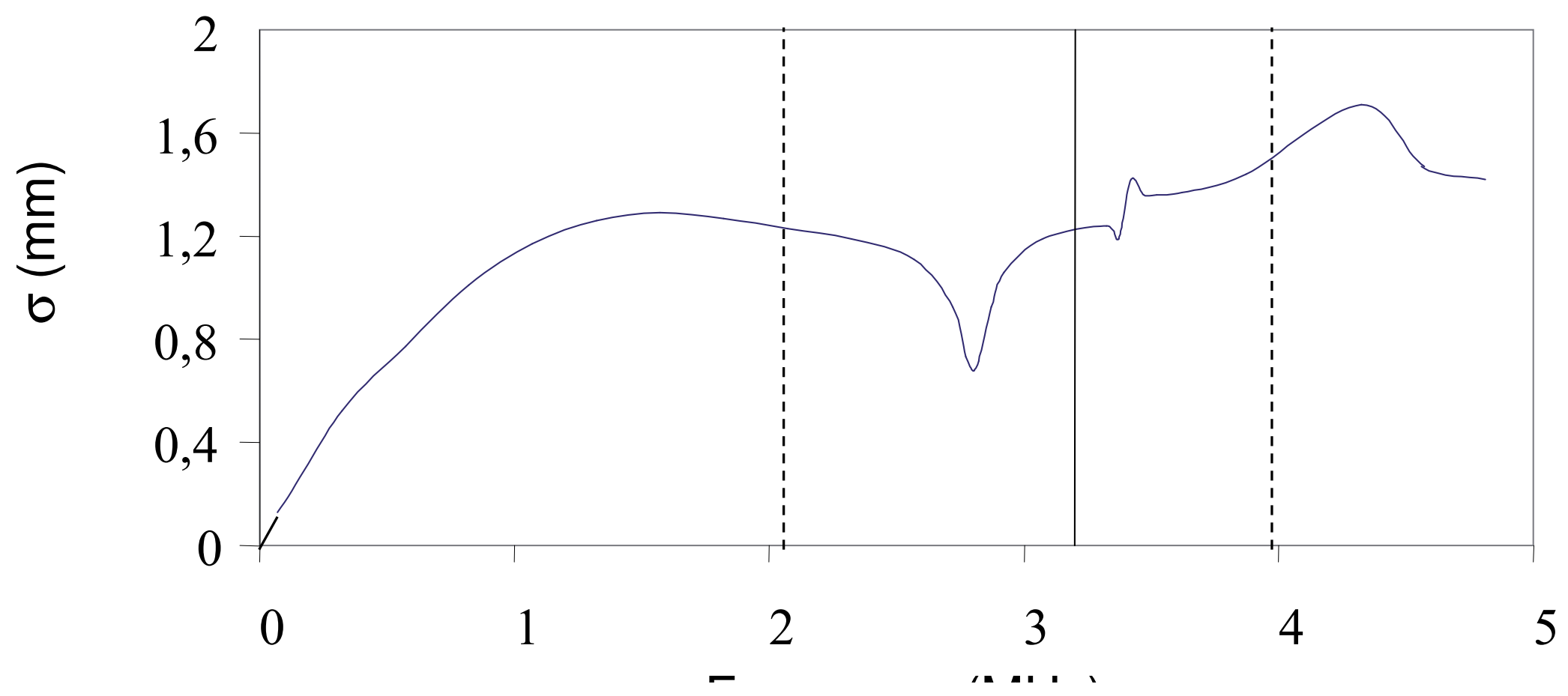




\section{Scattering from a single inclusion}

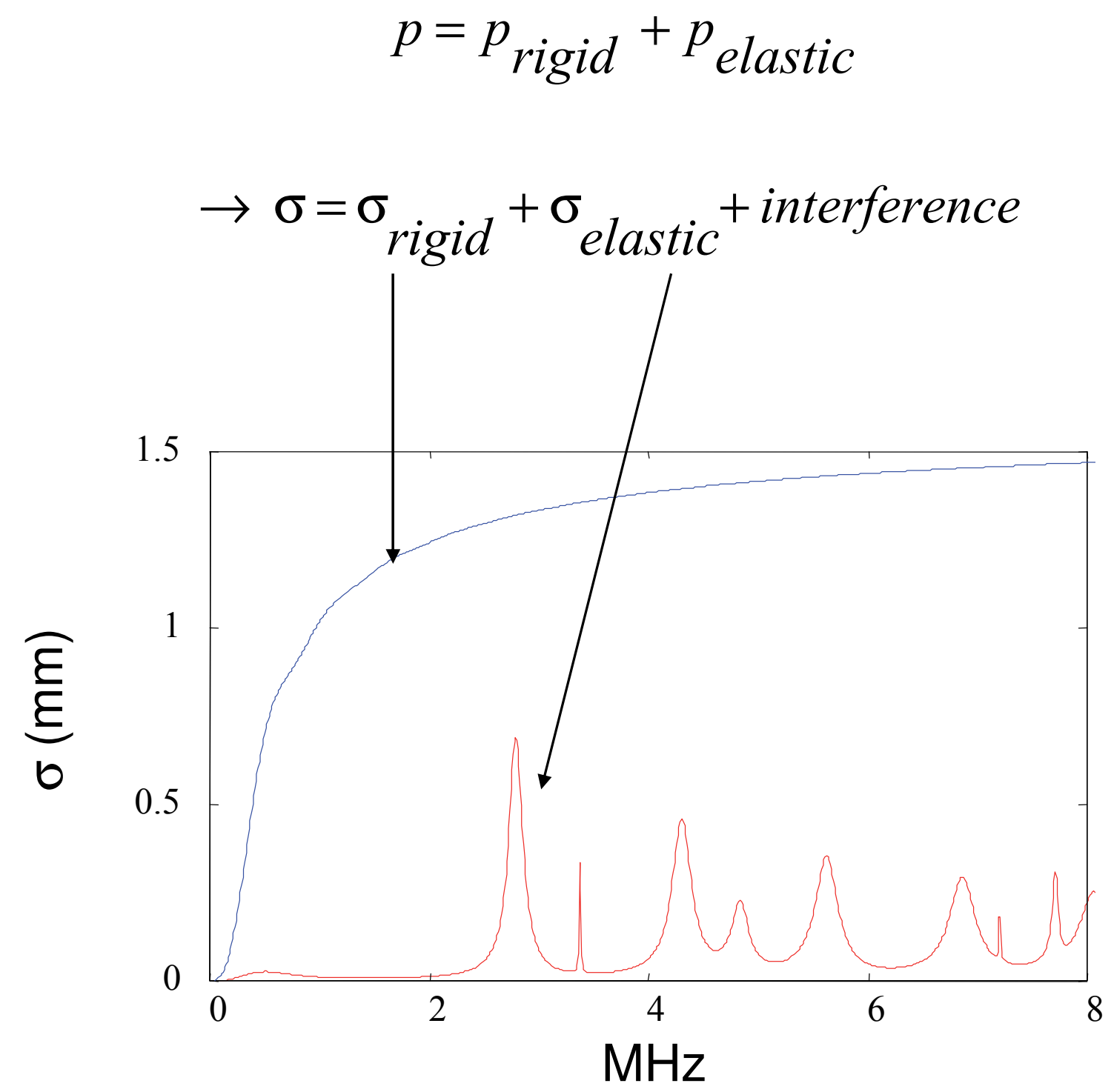




\section{The incoherent wave}
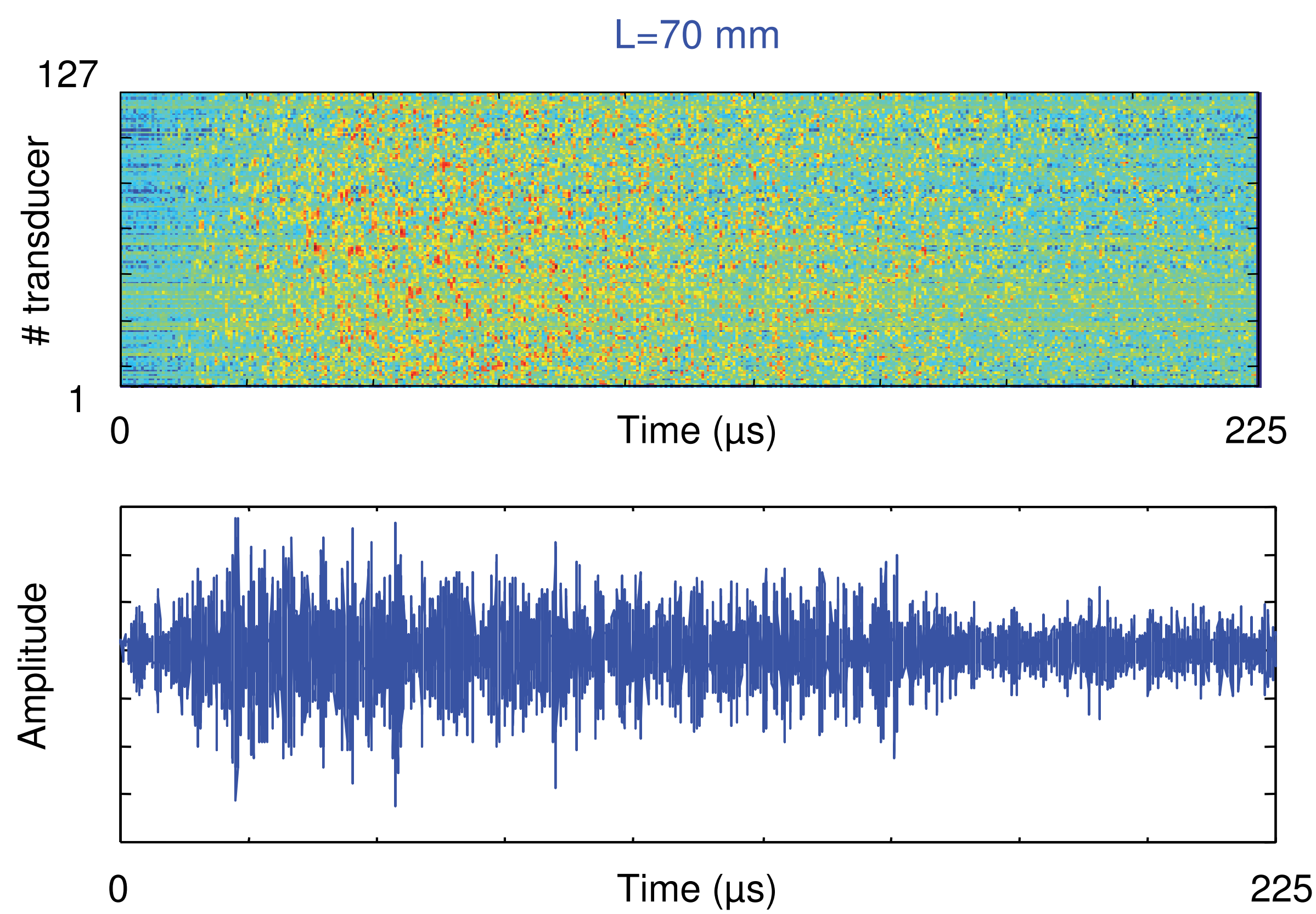


\section{The Bethe Salpeter equation}

The incoherent intensity $\quad \mathrm{I}_{\text {incoh }}=\left\langle\left|\mathrm{G}\left(\mathbf{r}, \mathbf{r}_{0} ; \mathrm{t}\right)\right|^{2}\right\rangle$

$$
\begin{array}{r}
\left\langle\mathrm{G}\left(\mathbf{r}, \mathbf{r}_{0} ; \omega-\Omega / 2\right) \mathrm{G}^{*}\left(\mathbf{r}^{\prime}, \mathbf{r}_{0}^{\prime} ; \omega+\Omega / 2\right)\right\rangle=\left\langle\mathrm{G}\left(\mathbf{r}, \mathbf{r}_{0} ; \omega-\Omega / 2\right)\right\rangle\left\langle\mathrm{G}^{*}\left(\mathbf{r}^{\prime}, \mathbf{r}_{0}^{\prime} ; \omega+\Omega / 2\right)\right\rangle+ \\
\left.\int\left\langle\mathrm{G}\left(\mathbf{r}, \mathbf{r}_{1} ; \omega-\Omega / 2\right)\right\rangle \mathrm{G}^{*}\left(\mathbf{r}^{\prime}, \mathbf{r}_{2} \omega+\Omega / 2\right)\right\rangle \mathrm{U}\left(\mathbf{r}_{1}, \mathbf{r}_{2}, \mathbf{r}_{3}, \mathbf{r}_{4}\right)\left\langle\mathrm{G}\left(\mathbf{r}_{3}, \mathbf{r}_{0} ; \omega-\Omega / 2\right) \mathrm{G}^{*}\left(\mathbf{r}_{4}, \mathbf{r}_{0}^{\prime} ; \omega+\Omega / 2\right)\right\rangle \mathrm{dr}_{1} \mathrm{~d} \mathbf{r}_{2} \mathrm{~d} \mathbf{r}_{2} \mathrm{~d} \mathbf{r}_{4}
\end{array}
$$

- Boltzmann approximation $\Rightarrow$ Transfer radiative equation for the specific intensity

- Diffusion approximation $\Rightarrow$ Diffusion equation $\frac{\partial \mathrm{I}_{\text {incoh }}}{\partial \mathrm{t}}=\mathrm{D} \Delta \mathrm{I}_{\text {incoh }} \quad \mathrm{D}=\frac{1}{3} \mathrm{~V}_{\mathrm{E}} \ell^{*}$ 


\section{The incoherent wave}

$\square$ Time-of-flight distribution $\quad \mathrm{I}_{\text {incoh }}=\left\langle|\mathrm{G}|^{2}\right\rangle$

$$
\begin{aligned}
& \mathrm{D}=3.2 \mathrm{~mm}^{2} / \mu \mathrm{s} \\
& \ell^{*}=4.8 \mathrm{~mm} \\
& \ell_{\mathrm{a}}=350 \mathrm{~mm}\left(\tau_{\mathrm{a}}=250 \mu \mathrm{s}\right)
\end{aligned}
$$

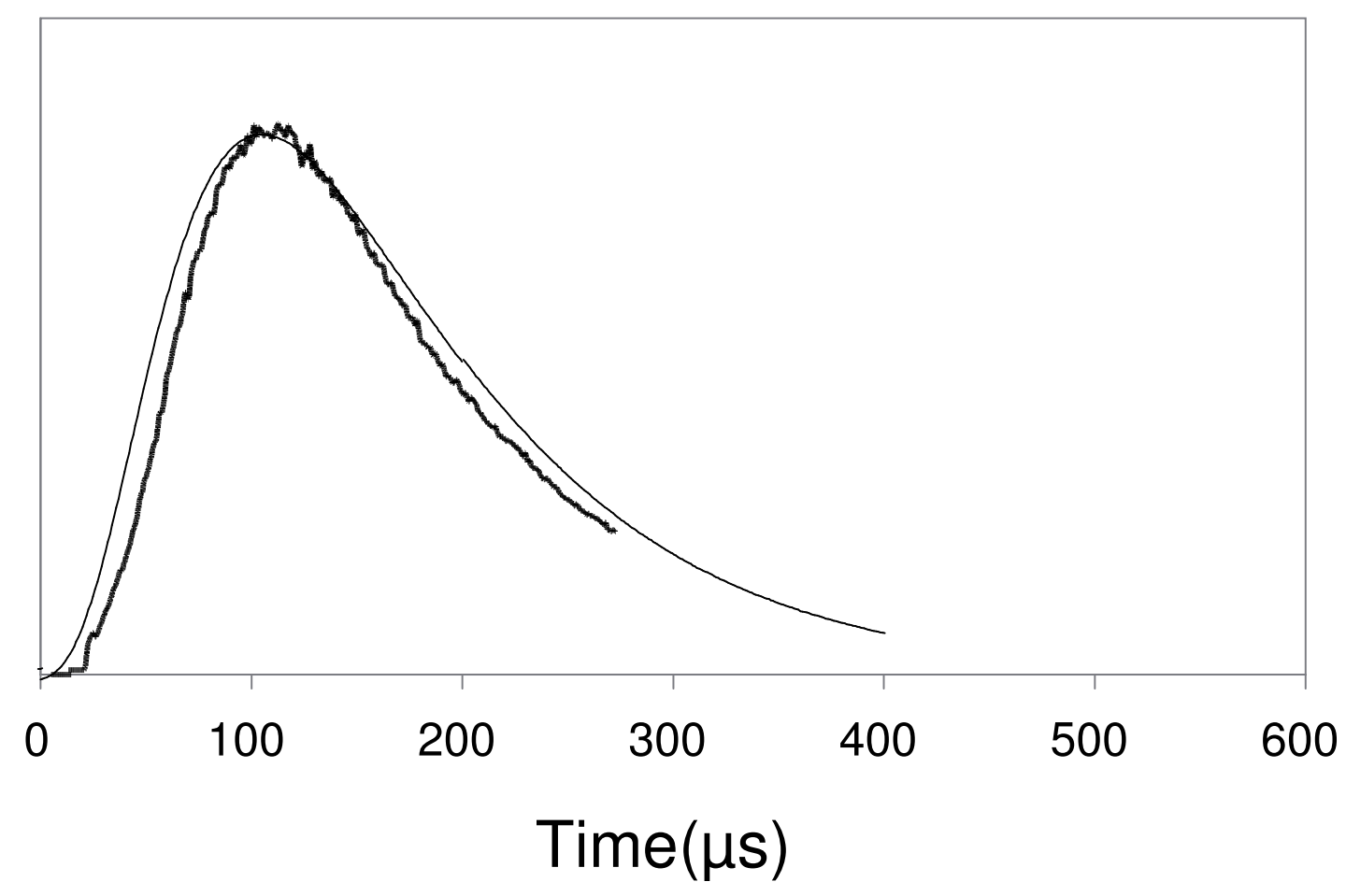

$\square$ Total transmission varies as $\sim /$ L (ohm's law) 


\section{Beyond the diffusion approximation}

\section{The backscattering cone}

M.P. van Albada and A. Lagendijk, Phys. Rev. Lett. 55, 2692 (1985)

E. Wolf and G. Maret, Phys. Rev. Lett. 55, 2696 (1985)

A. Tourin, B. A. Van Tiggelen, A. Derode, P. Roux, M. Fink,, Phys. Rev. Lett 79, 3637 (1997)

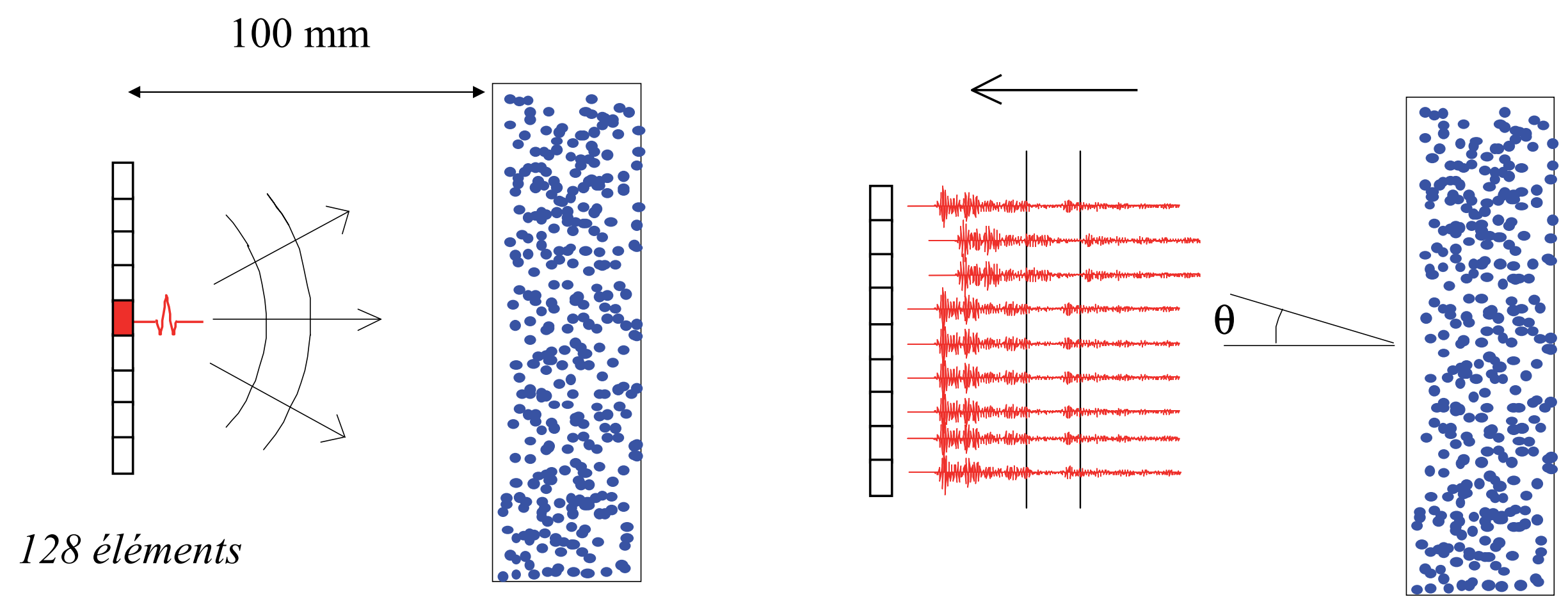

EMISSION

RECEPTION 


\section{The backscattering cone}

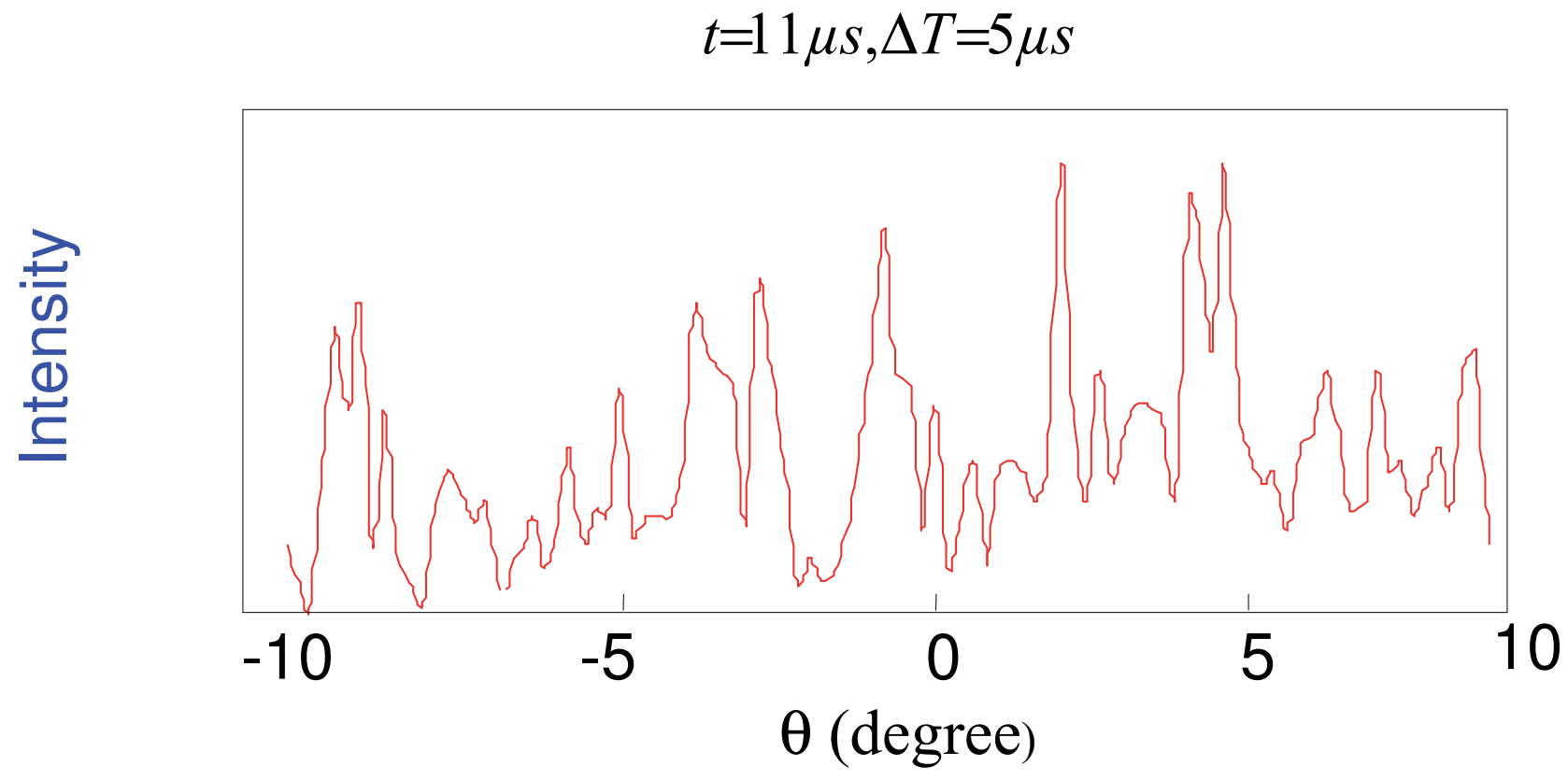

\section{Speckle}

One realisation

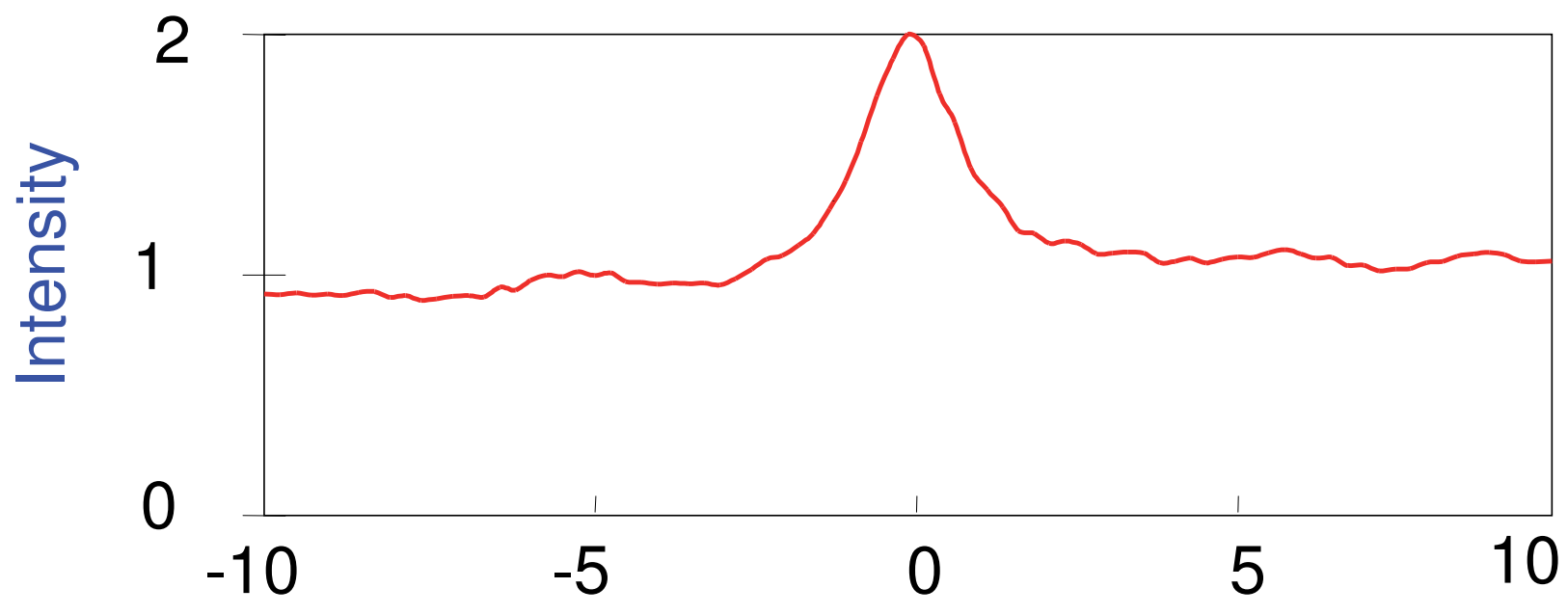

Averaging over 80 realisations 


\section{The backscattering cone}

$$
I=|A|^{2}=\sum_{i, j} A_{i} A_{j}^{*}
$$

Speckle

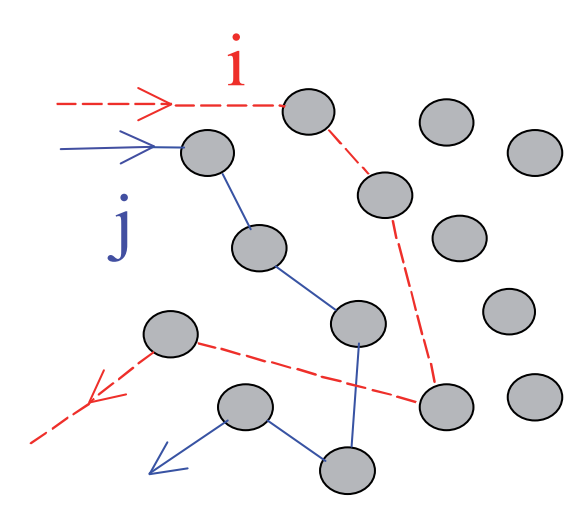

Incoherent contribution

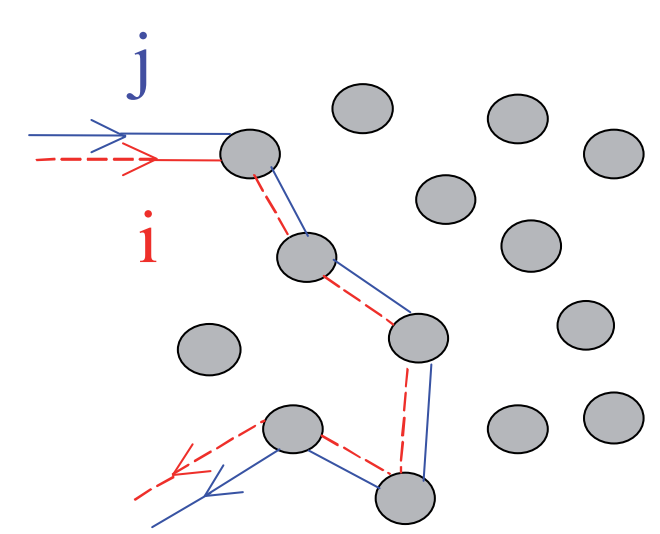

Coherent contribution

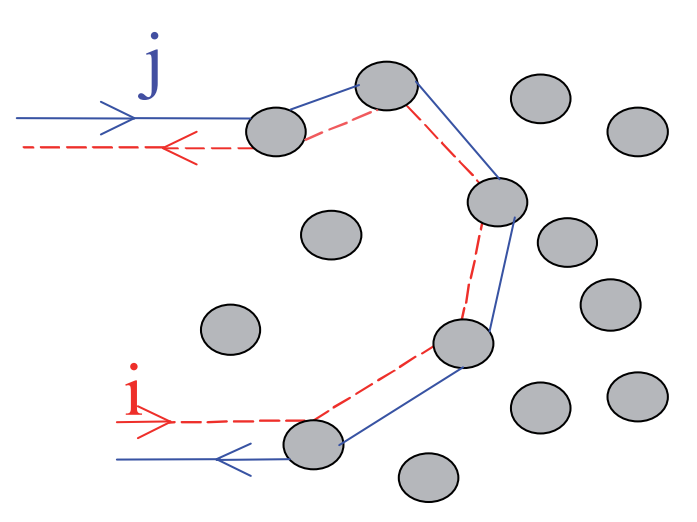




\section{The backscattering cone}

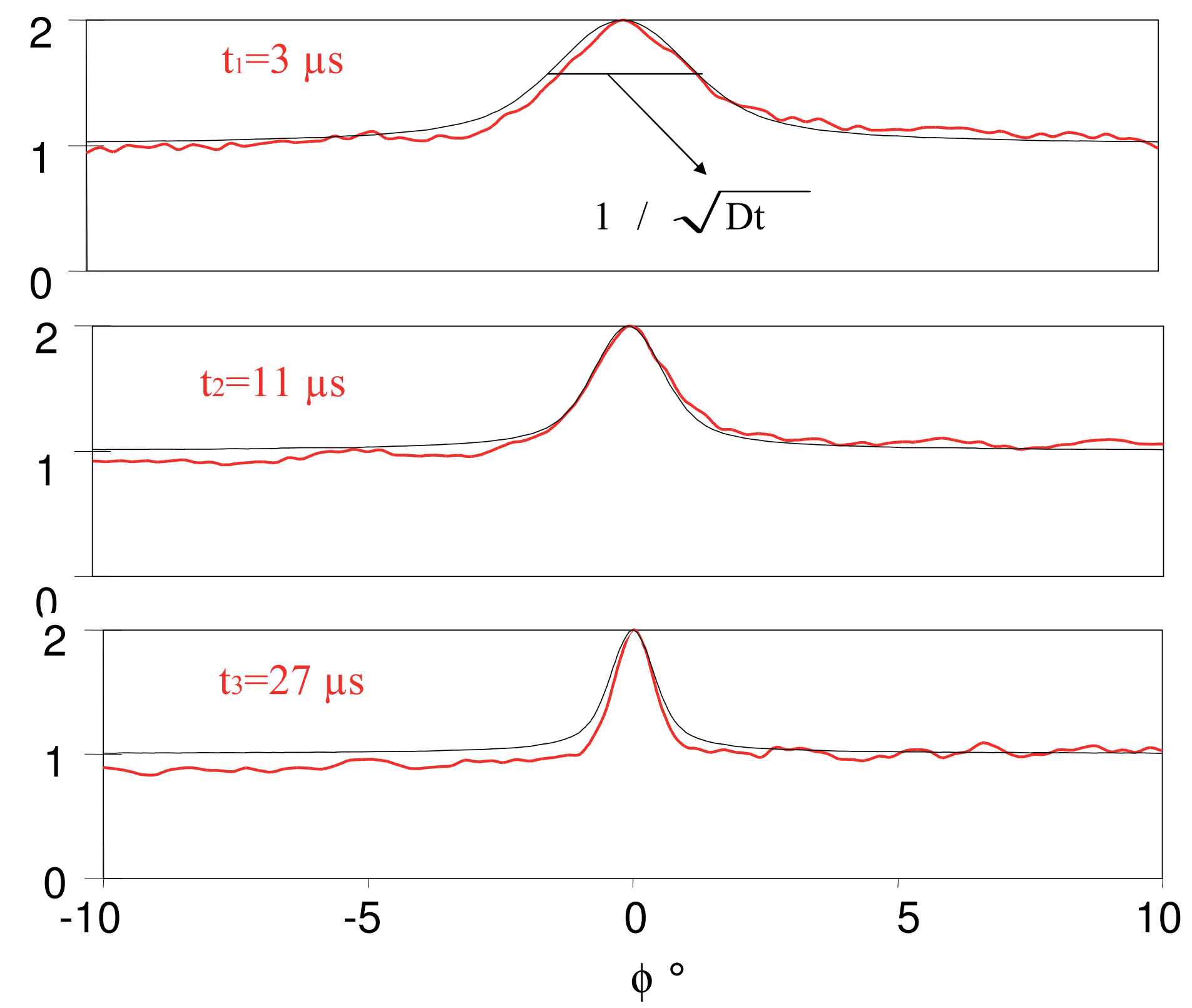




\section{The backscattering cone}

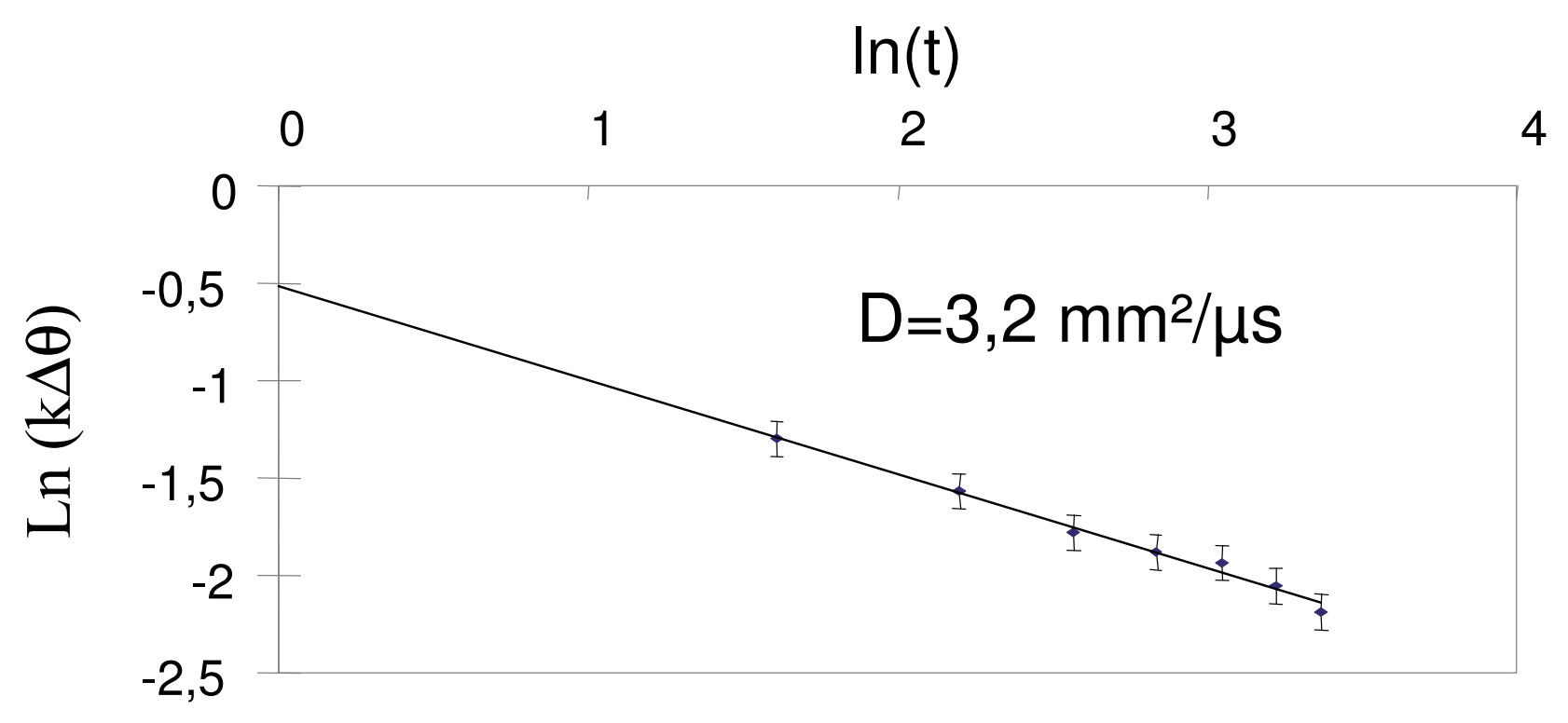

Dynamic CBE

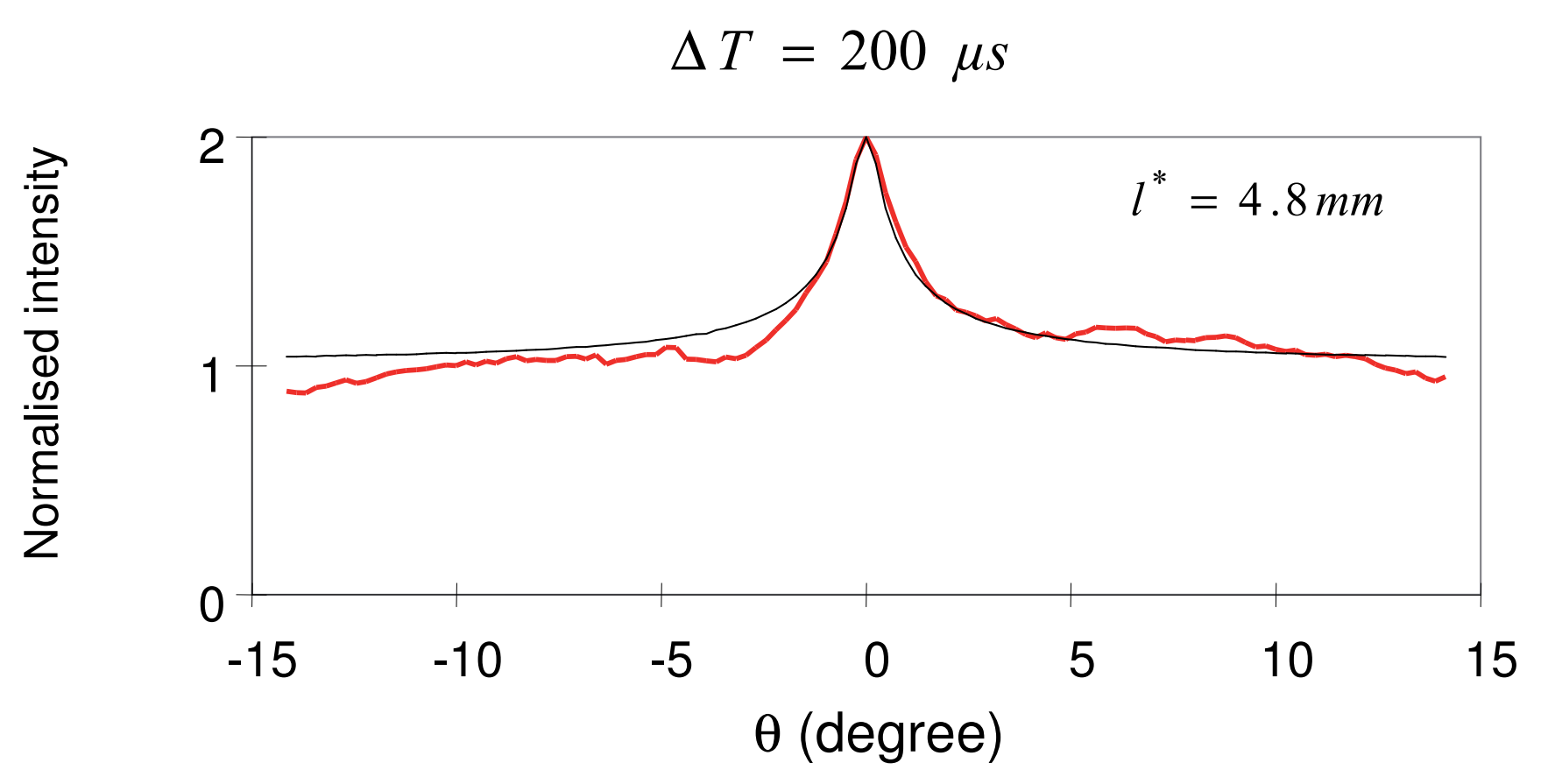

Stationary CBE 


\section{Weak localisation}

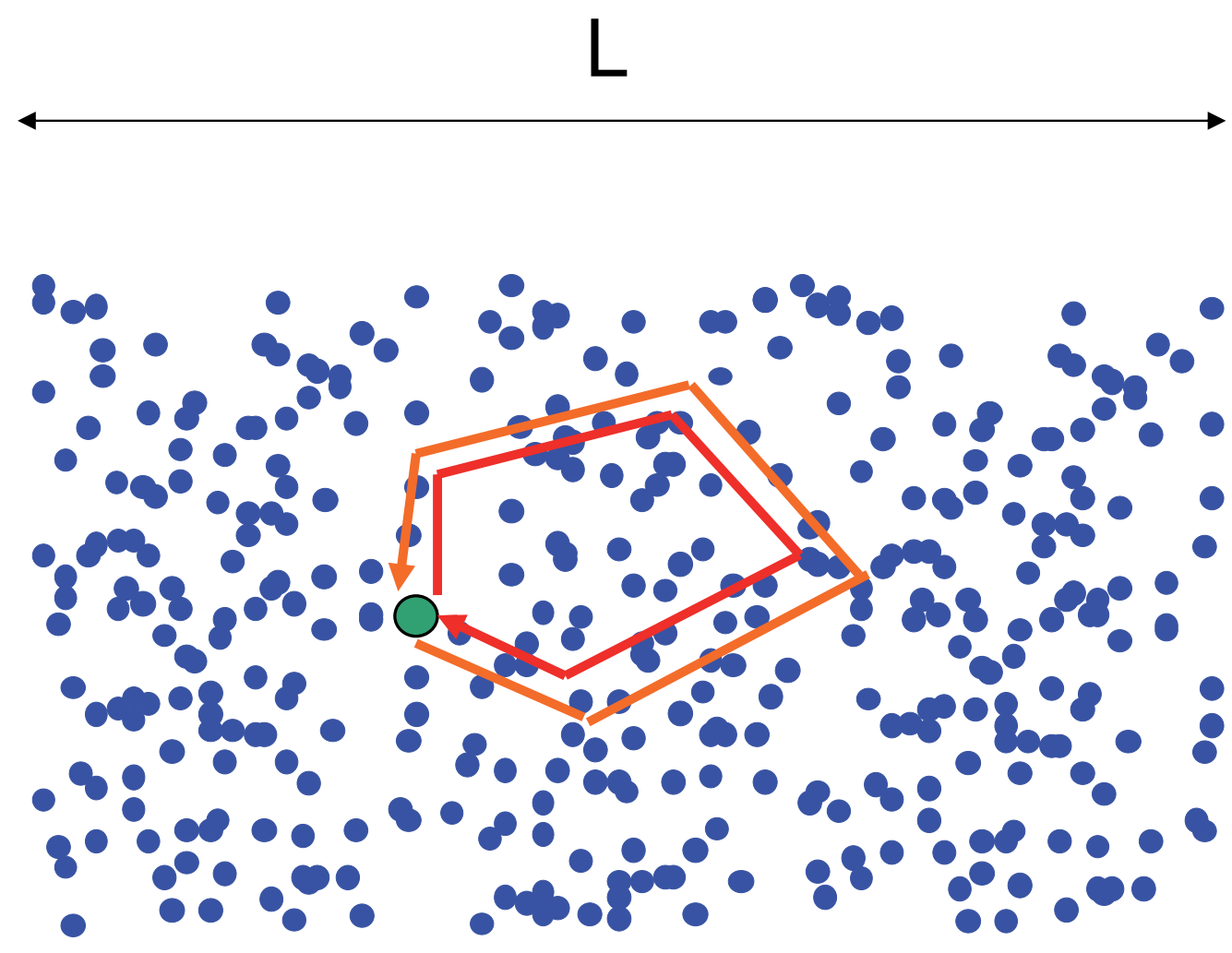




\section{Using disorder}

for controlling wave propagation 


\section{A random ffber}

H. De Raedt, Ad Lagendijk, Pedro de Vries

«Transverse localization of light »

Phys. Rev. Lett. 62, 47 (1989)

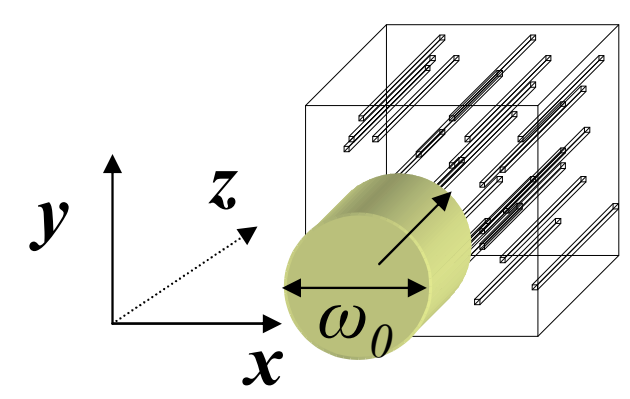

$$
\left\{\begin{array}{c}
H \equiv \frac{\partial^{2}}{\partial x^{2}}+\frac{\partial^{2}}{\partial y^{2}}+V(x, y) \\
V(x, y) \equiv k^{2}\left(n^{2}(x, y)-n_{0}^{2}\right)
\end{array}\right.
$$

Lee,P. A. et al., Rev. Mod. Phys. 57, 287-337 (1985)

$$
\xi_{l o c}=l^{*} \exp \left(\frac{\pi}{2} k_{F} l^{*}\right) \quad k_{F} \equiv k_{T} \propto \omega_{0}^{-1}
$$




\section{A random fiber}

Schwartz et al., Nature 446, 52-55 (2007)

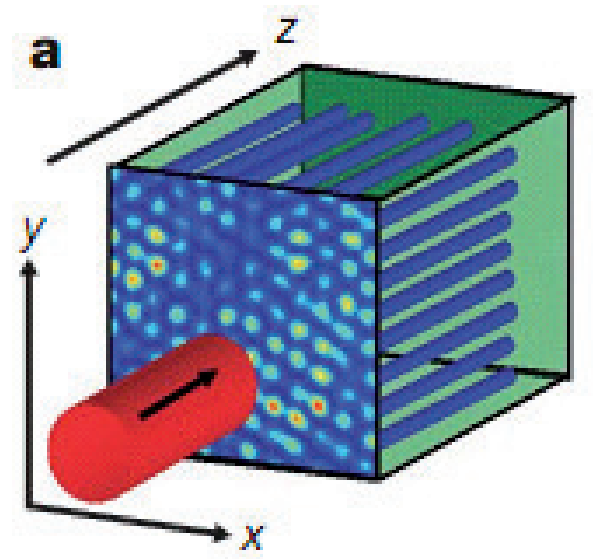

Intensity distribution after $\mathrm{L}=10 \mathrm{~mm}$ propagation

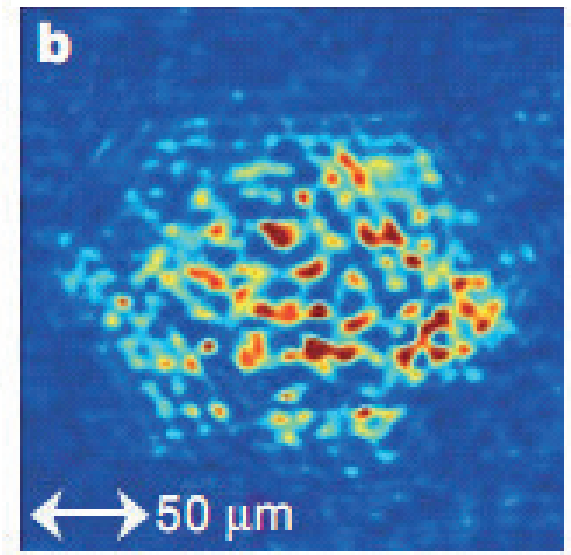

c

hexagonal lattice $15 \%$ positional disorder (average over 100 realizations) 


\section{A random fiber}

Alice Bretagne, AT

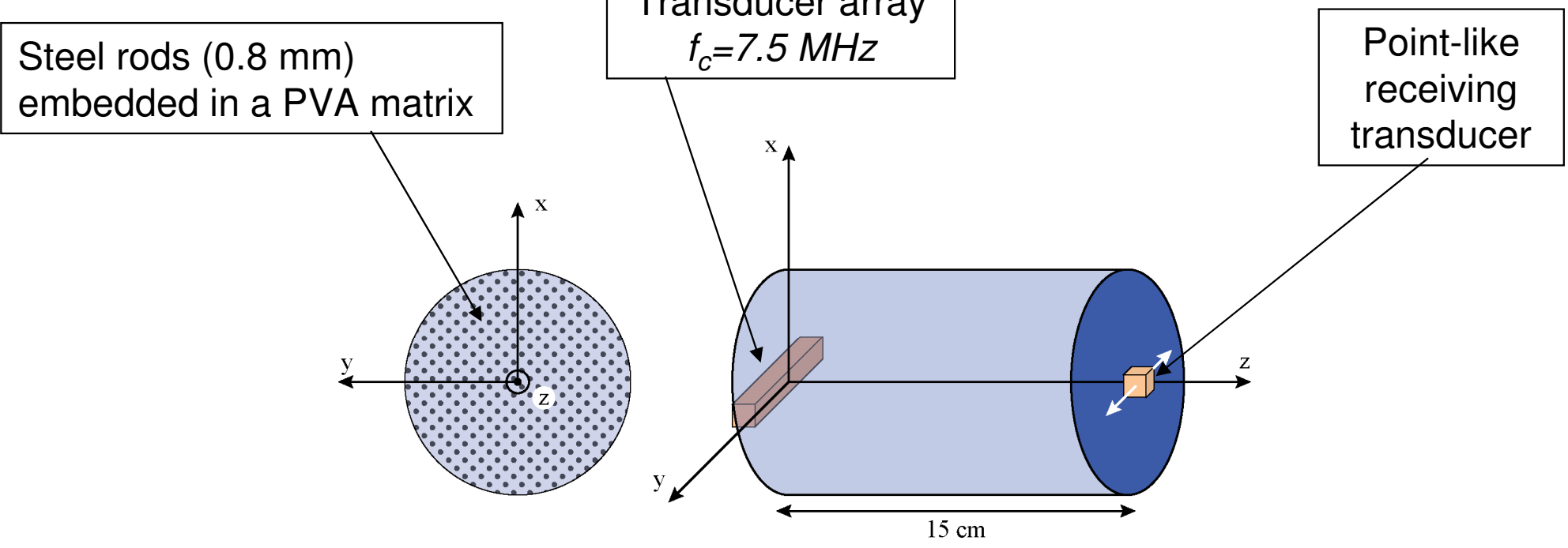

Ordered sample

(triangular lattice, a=2.4mm)

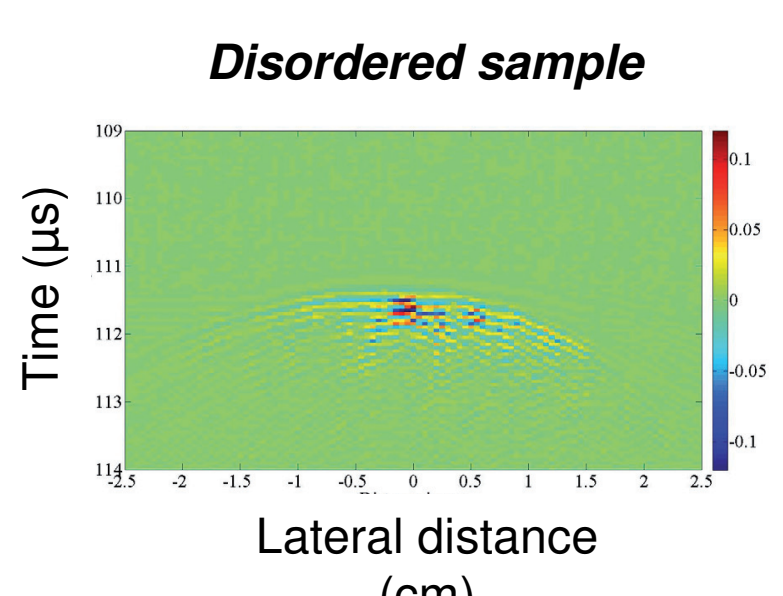

Ordered (blue)

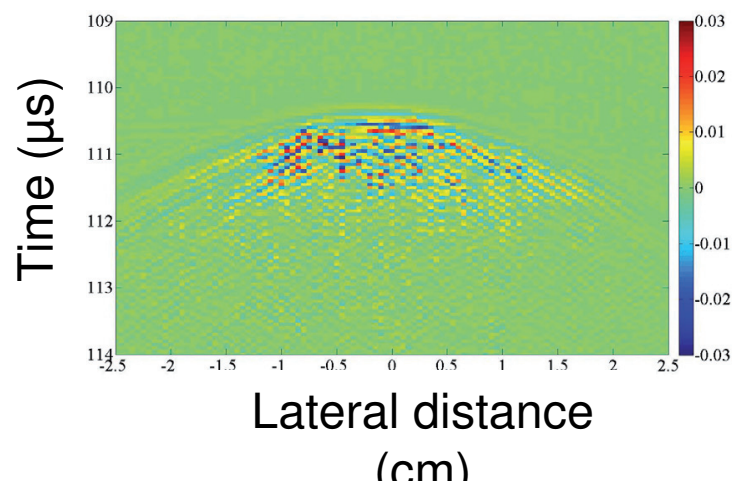

(cm)

(cm)

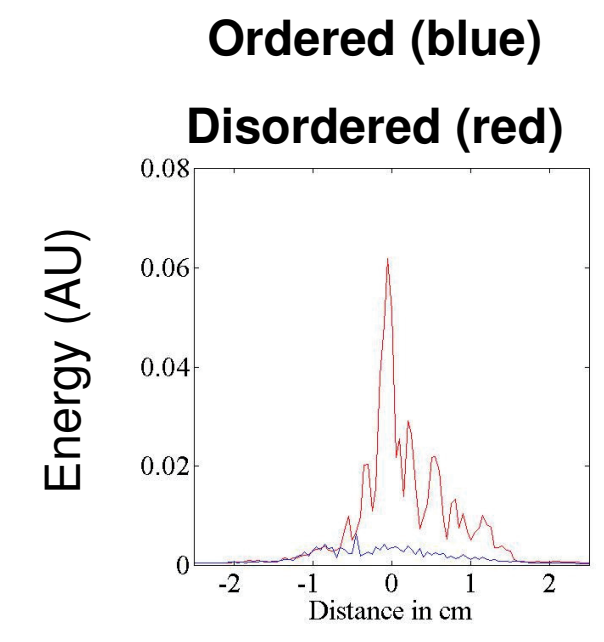




\section{A random lens}
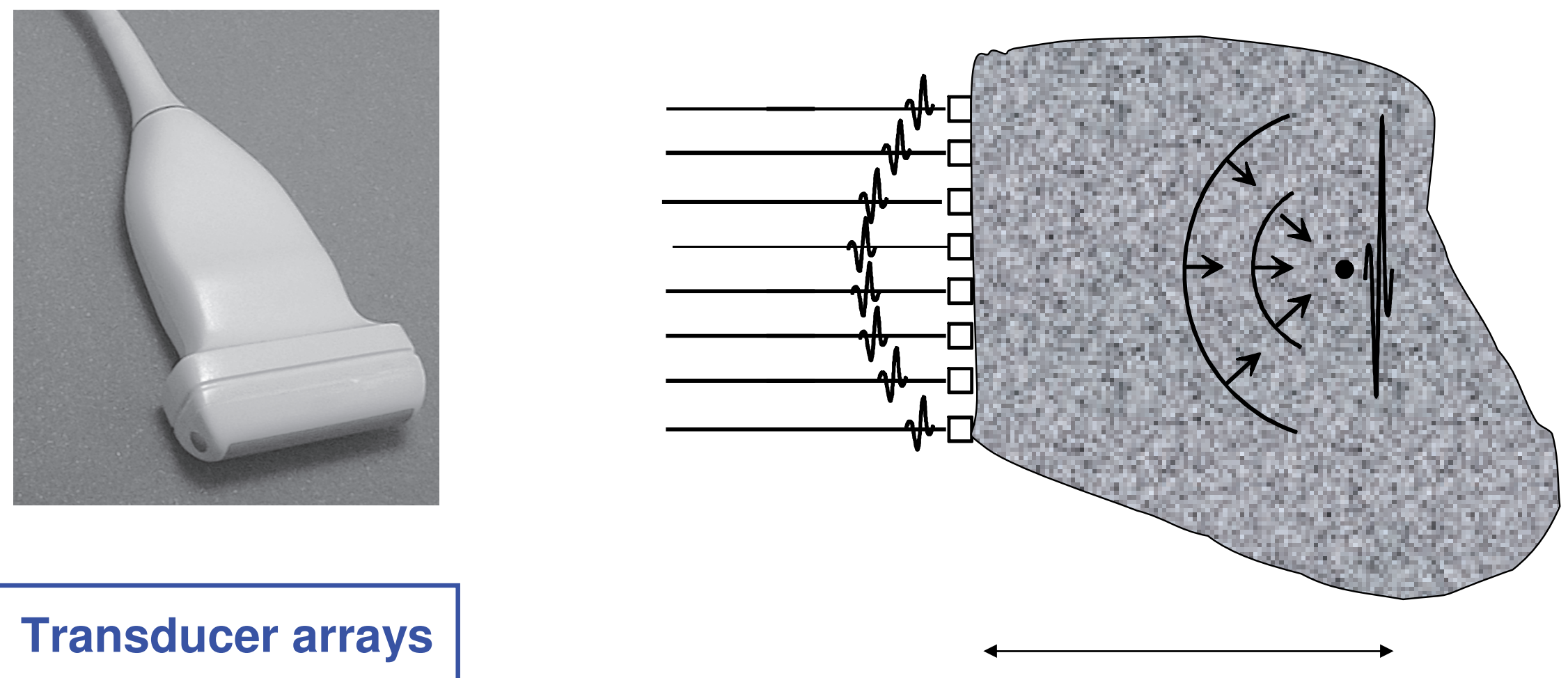

Transducer arrays

$\mathrm{f} \sim 500 \mathrm{kHz}$ à $5 \mathrm{MHz}$ $\lambda \sim \mathrm{mm}$

$\mathrm{F}<\ell_{\mathrm{s}}$ 


\section{A random lens}

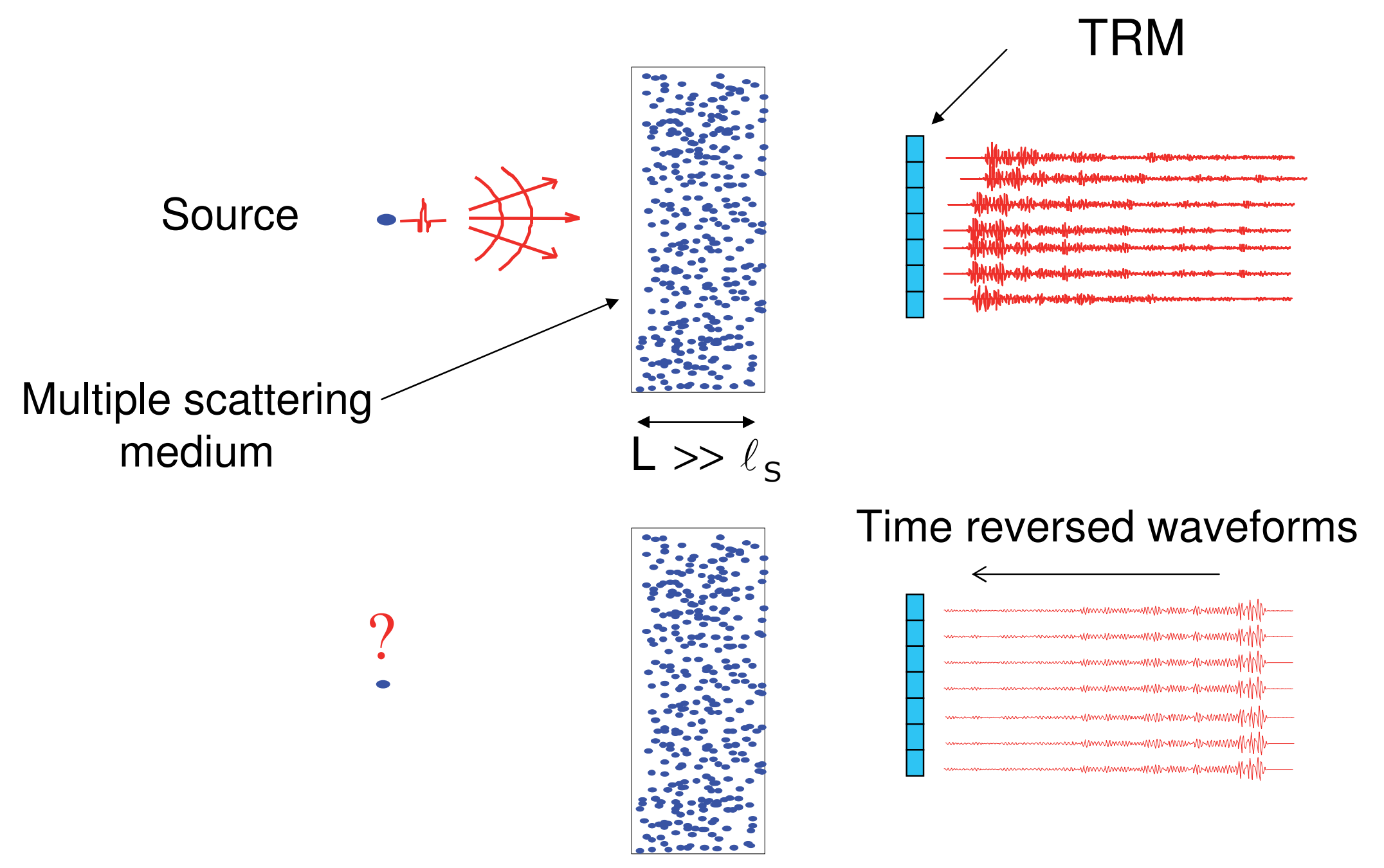

A.Derode, A. Tourin, P. Roux, M. Fink 


\section{A random lens}

Waveform transmitted in water and received at transducer \# 64

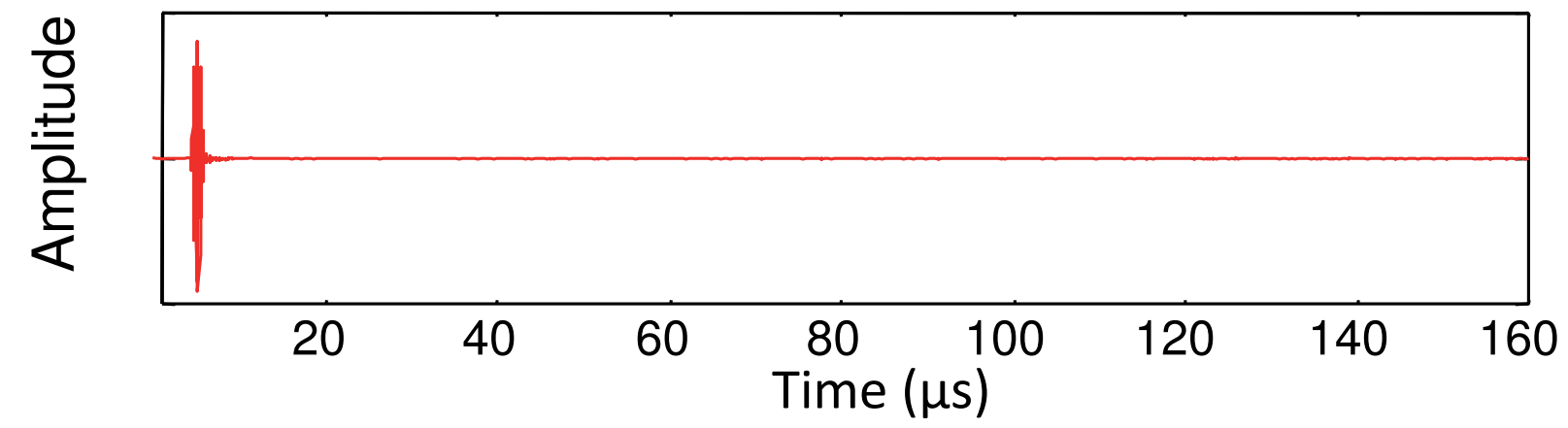

Waveform transmitted through the rods and received at transducer \#64

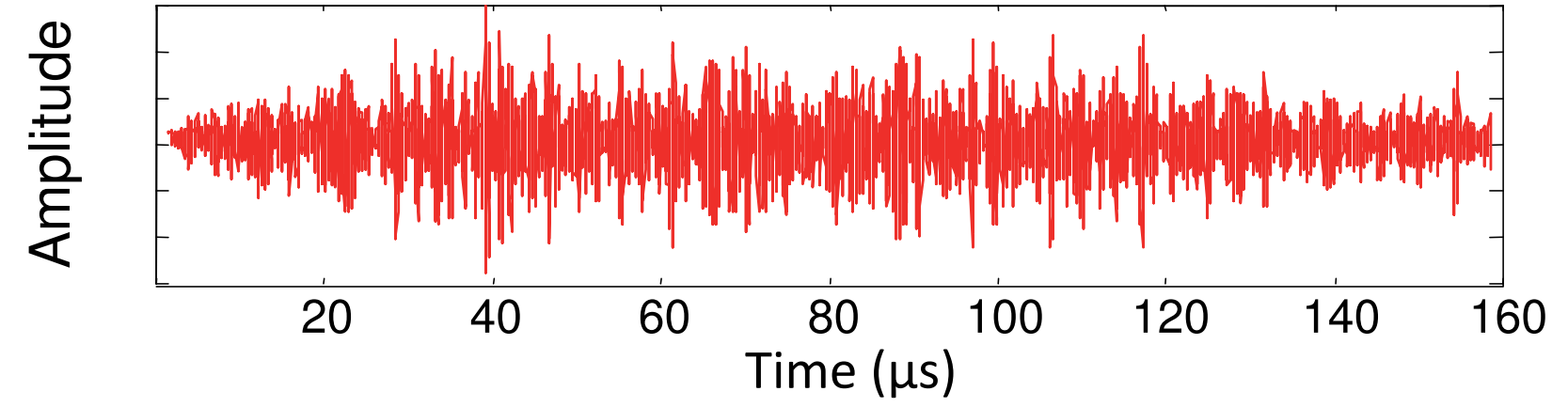

Time reversed wave recorded at the source location

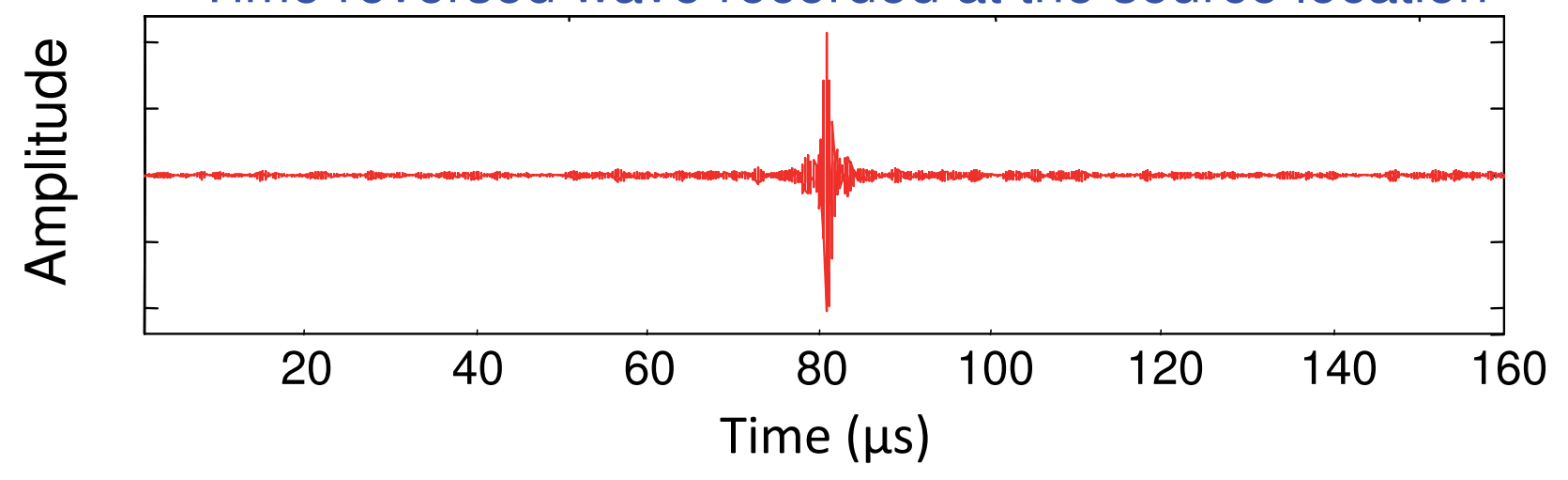




\section{A random lens}

\section{Spatial Focusing : the hyperfocusing effect}

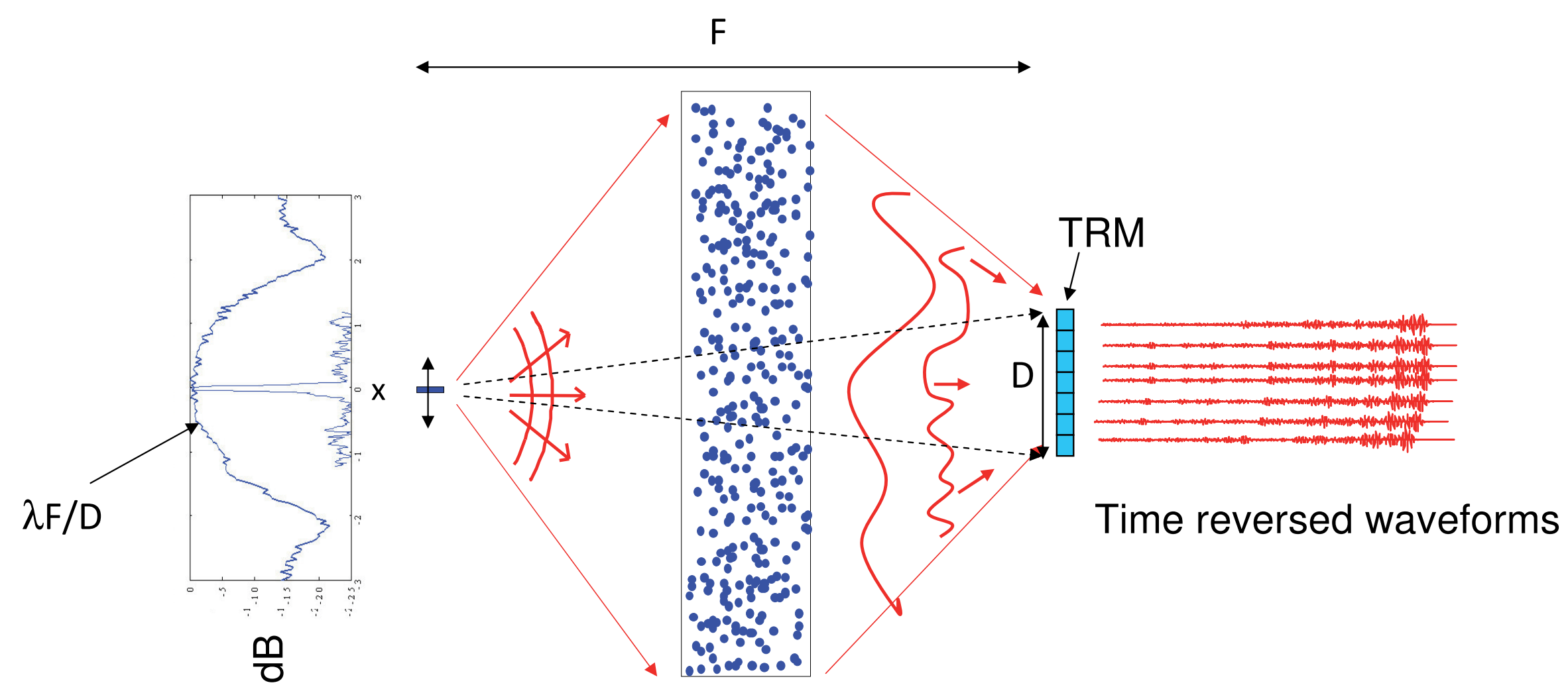

Beamwidth at $-12 \mathrm{~dB}: 1 \mathrm{~mm}$ (scattering medium) / $35 \mathrm{~mm}$ (free space)

Spatial resolution does not depend on the array aperture ! 


\section{A random lens}

\section{One-channel time reversal mirror}

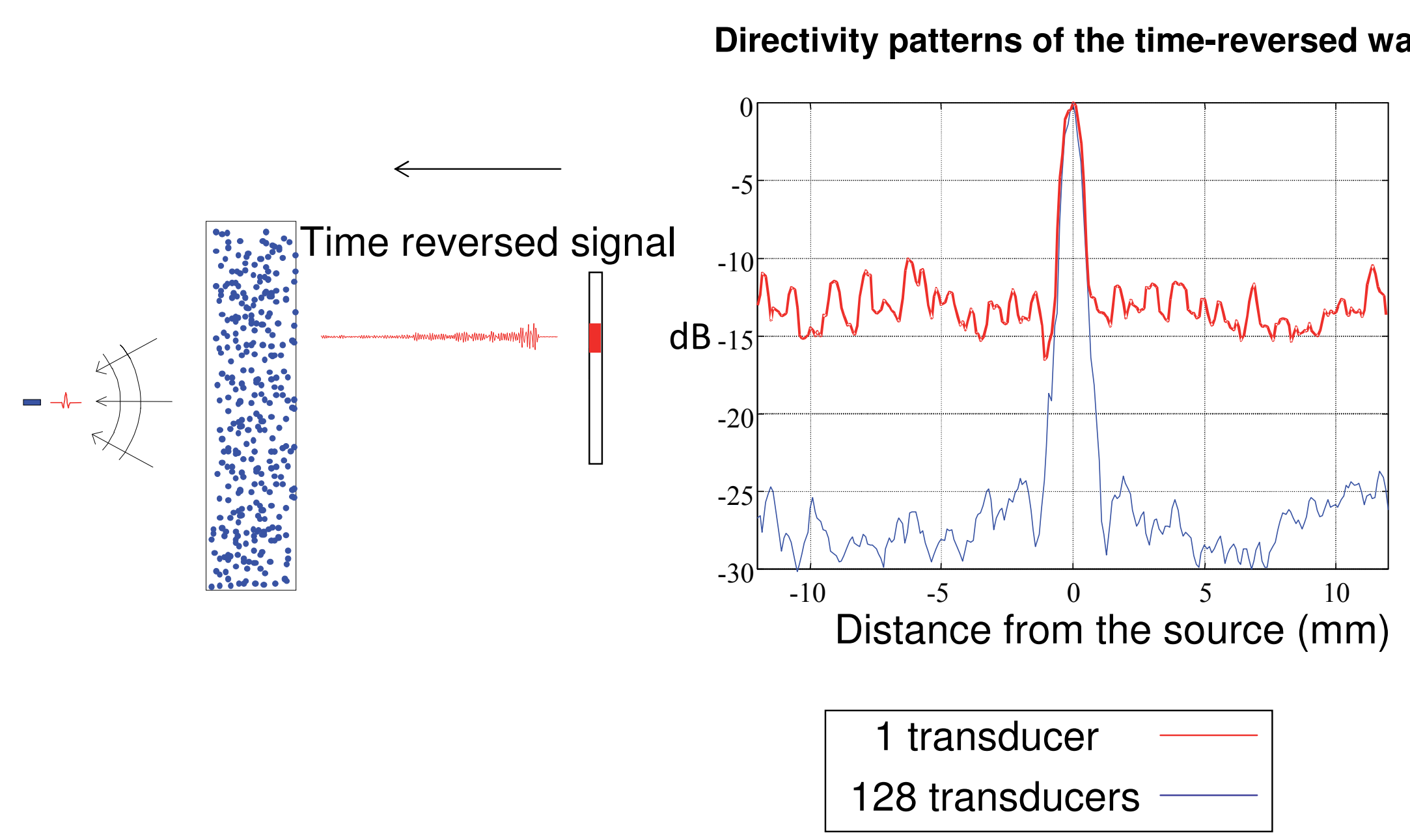




\section{A random lens}

\section{An alternative approach : wave front shaping}

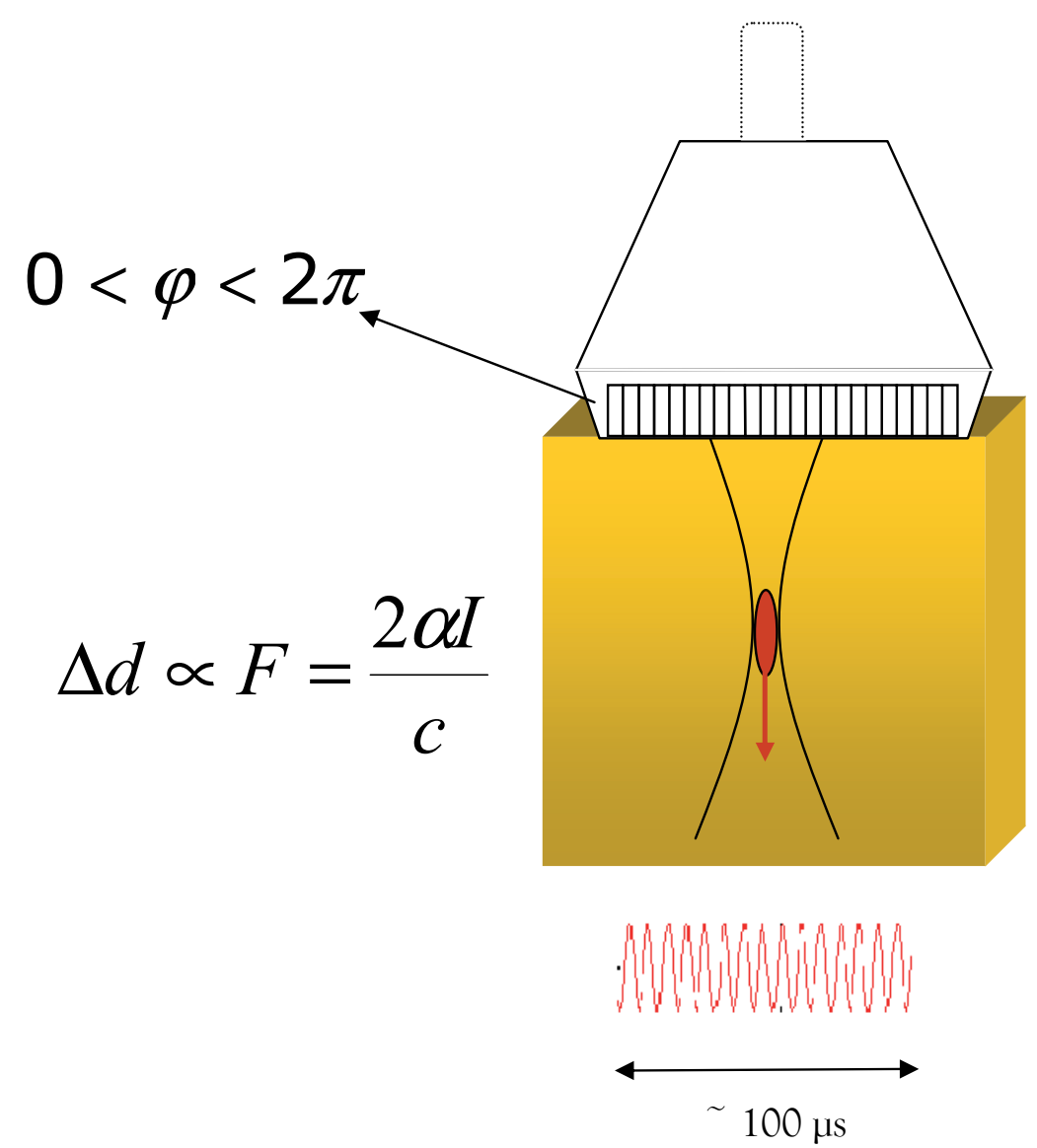

- I.M. Vellekoop and A.P. Mosk Optics Lett. 32, 2309 (2007)

- E. Herbert, M. Pernot, M. Tanter, G. Montaldo, M. Fink IEEE transactions on ultrasonics, ferroelectrics, and frequency control 56 (11), 2388 (2009)

Two problematic issues

low sensitivity (one by one) a lot of iterations are needed 


\section{A random lens}

In the case of two transducers, the intensity at the intended focus is :

$$
\begin{aligned}
& I=\frac{P_{0}^{2}}{\rho C^{2}}\left[A_{1}^{2}+A_{2}^{2}+2 A_{1} A_{2} \cos \left(\Phi_{1}-\Phi_{2}\right)\right] \\
& \text { i.e., } \quad I=B+C \cos (\Phi)
\end{aligned}
$$

When a phase $\mathrm{x}$ is added to the 2 nd transducer, the intensity at focus is :

$$
\mathrm{I}(\mathrm{x})=\mathrm{B}+\mathrm{C} \cos (\Phi+\mathrm{x})
$$

At least 3 measurements must be performed

Finally, $A_{1}$ and $A_{2} e^{-i \Phi}$ are transmitted from the two transducers 


\section{A random lens}

Extension to a $\mathrm{N}$-transducer array using spatial coded excitations

$$
\begin{gathered}
\text { Hadamard matrix } \\
H=\frac{1}{2}\left(\begin{array}{cccccc}
1 & 1 & 1 & 1 & \ldots \\
1 & -1 & 1 & -1 & \\
1 & 1 & -1 & -1 & \\
1 & -1 & -1 & 1 & \\
\ldots & & & & \ldots
\end{array}\right)
\end{gathered}
$$

$$
\begin{aligned}
& \text { Transmission of } \\
& V_{0}(x, \omega)+e^{j x} \cdot V_{i}(x, \omega) \\
& (1<\mathrm{i}<\mathrm{N})
\end{aligned}
$$

Recording of $\mathrm{I}=\mathrm{I}(\mathrm{x}, \omega)$ at focus

Repeat the transmission for four different values

$$
(x=0, \pi, \pi / 2,-\pi / 2)
$$




\section{A random lens}

$$
\begin{aligned}
& \tan \left(\Psi_{\mathrm{i}}\right)=\frac{\mathrm{I}(\mathrm{x}=\pi / 2)-\mathrm{I}(\mathrm{x}=-\pi / 2)}{\mathrm{I}(\mathrm{x}=\pi)-\mathrm{I}(\mathrm{x}=0)} \\
& \mathrm{B}_{\mathrm{i}}=\frac{\sqrt{\mathrm{I}(0)+\mathrm{I}(\pi)-\mathrm{I}(\pi / 2)+\mathrm{I}(-\pi / 2)}}{2}
\end{aligned}
$$

$$
\left(\begin{array}{c}
A_{1} e^{j \Phi_{1}} \\
A_{2} e^{j \Phi_{2}} \\
\cdots \\
A_{i} e^{j \Phi_{i}} \\
A_{N} e^{j \Phi_{N}}
\end{array}\right)=H\left(\begin{array}{c}
B_{1} e^{j \Psi_{1}} \\
B_{2} e^{j \Psi_{2}} \\
\cdots \\
B_{i} e^{j \Psi_{i}} \\
B_{N} e^{j \Psi_{N}}
\end{array}\right)
$$




\section{A random lens}

Definition of spatio-temporal vectors

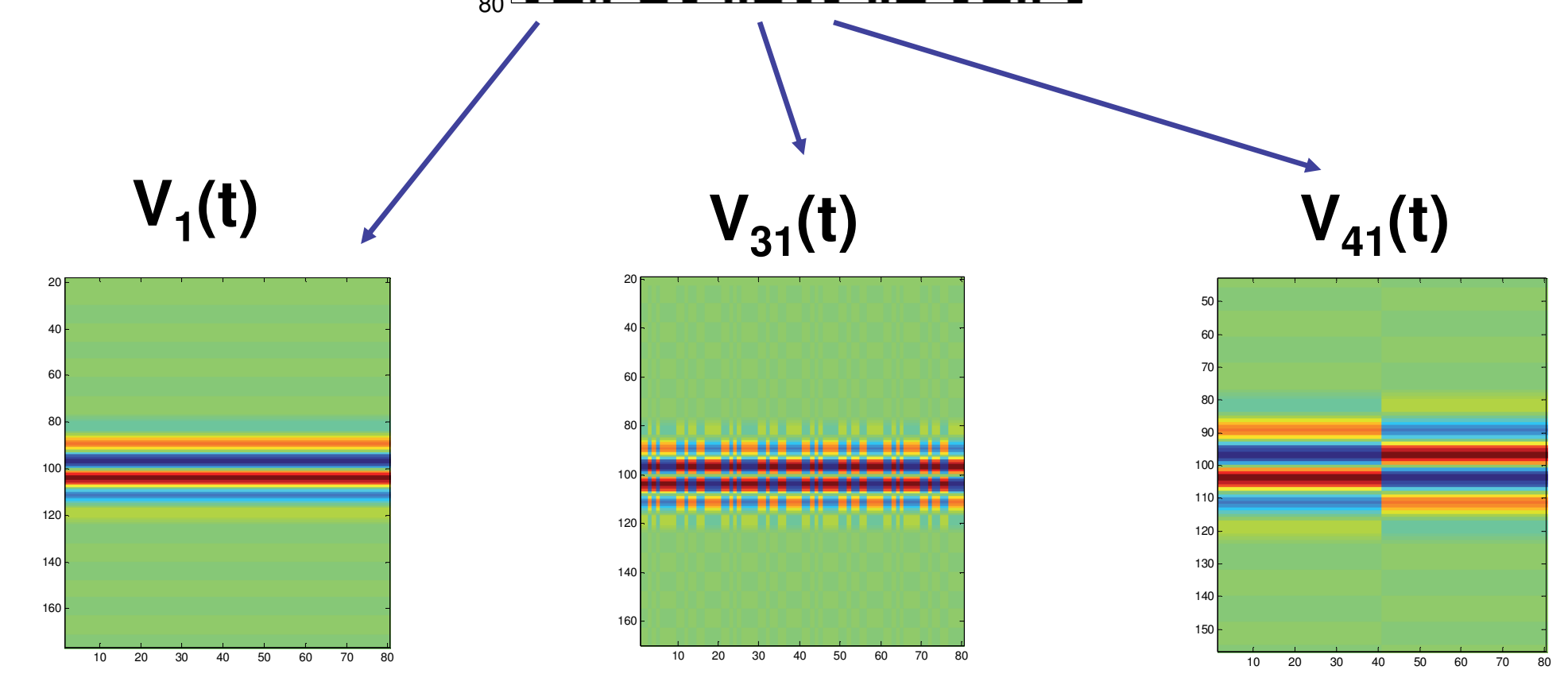




\section{A random lens}

Multiple scattering A. Bretagne, J. Aulbach, M. Tanter, M. Fink, A. Tourin medium
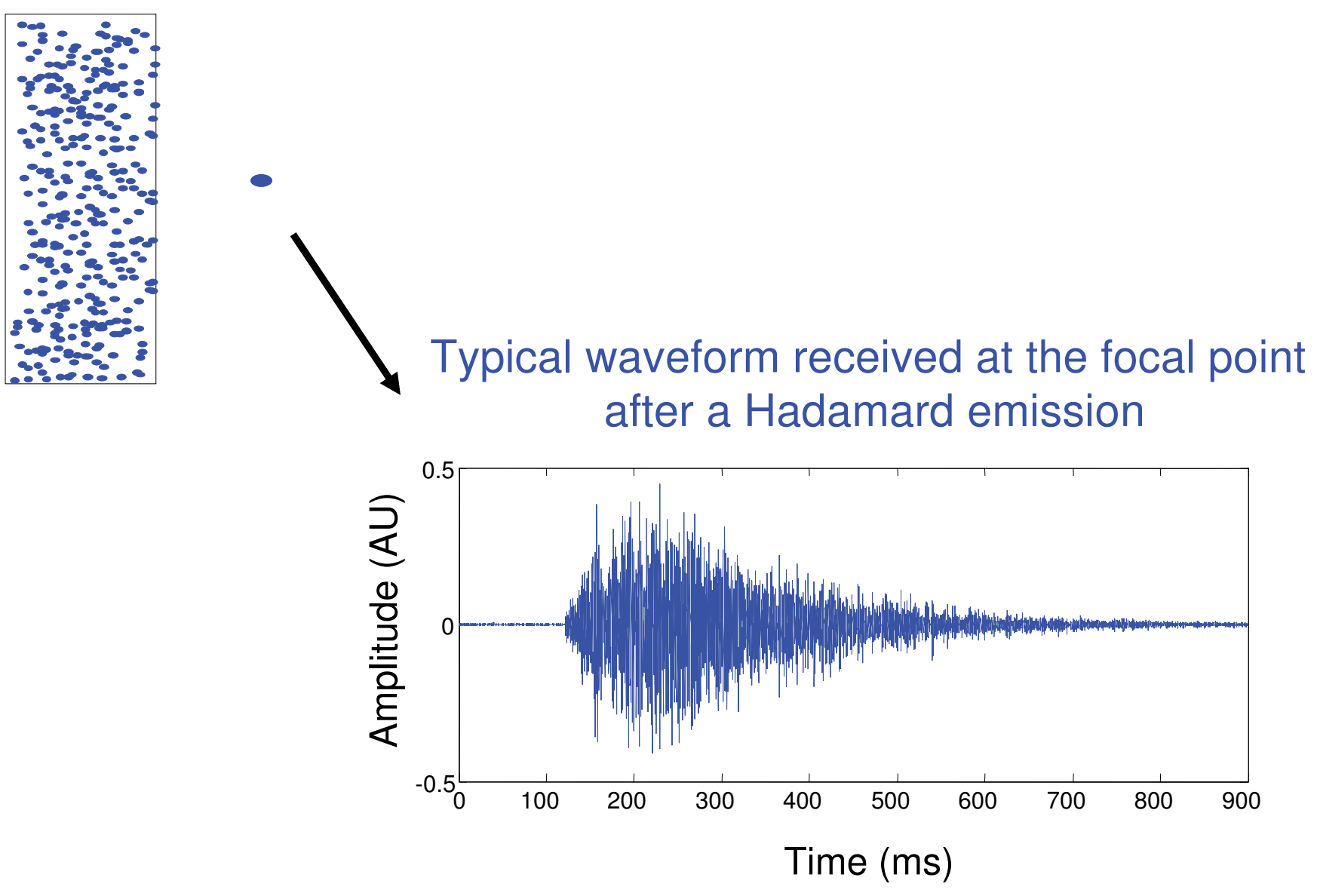


\section{A random lens}

\section{Monochromatic case}

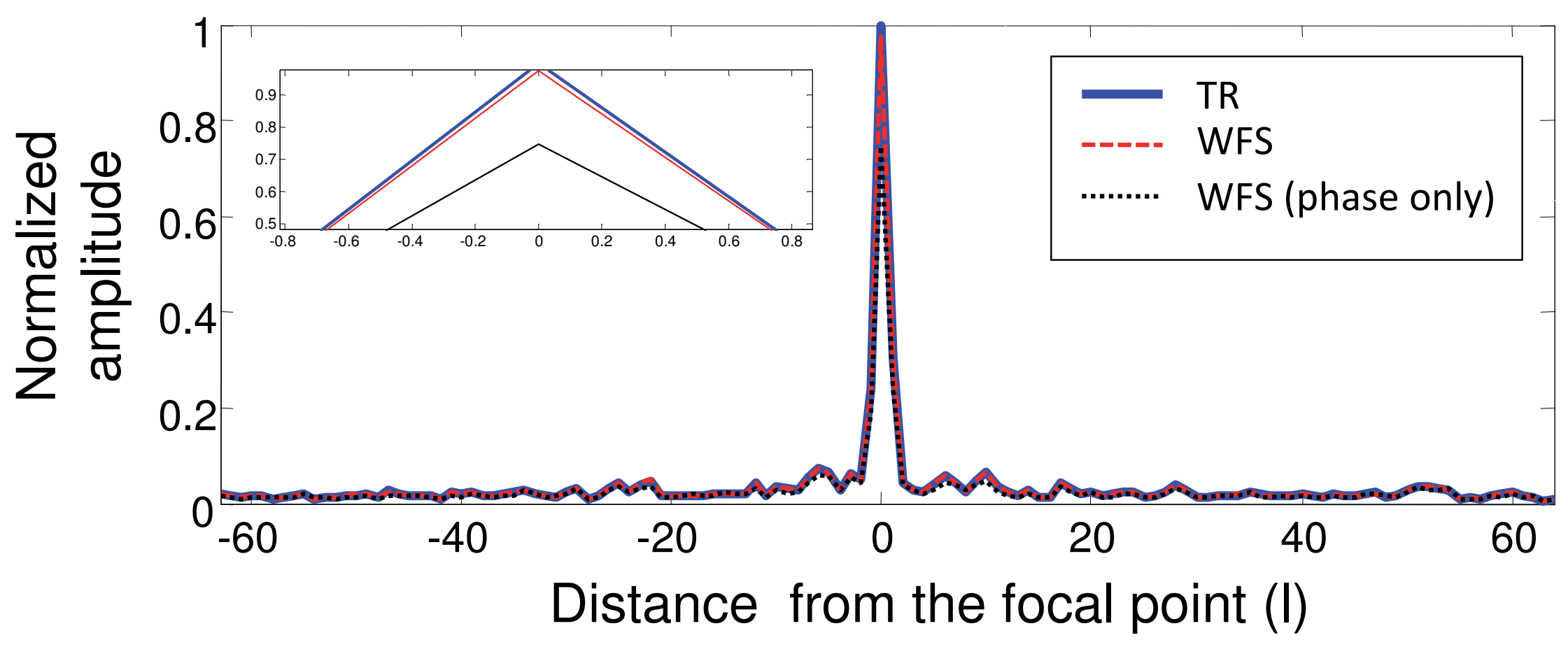




\section{A random lens}

\section{Broadband case}

Time Reversal

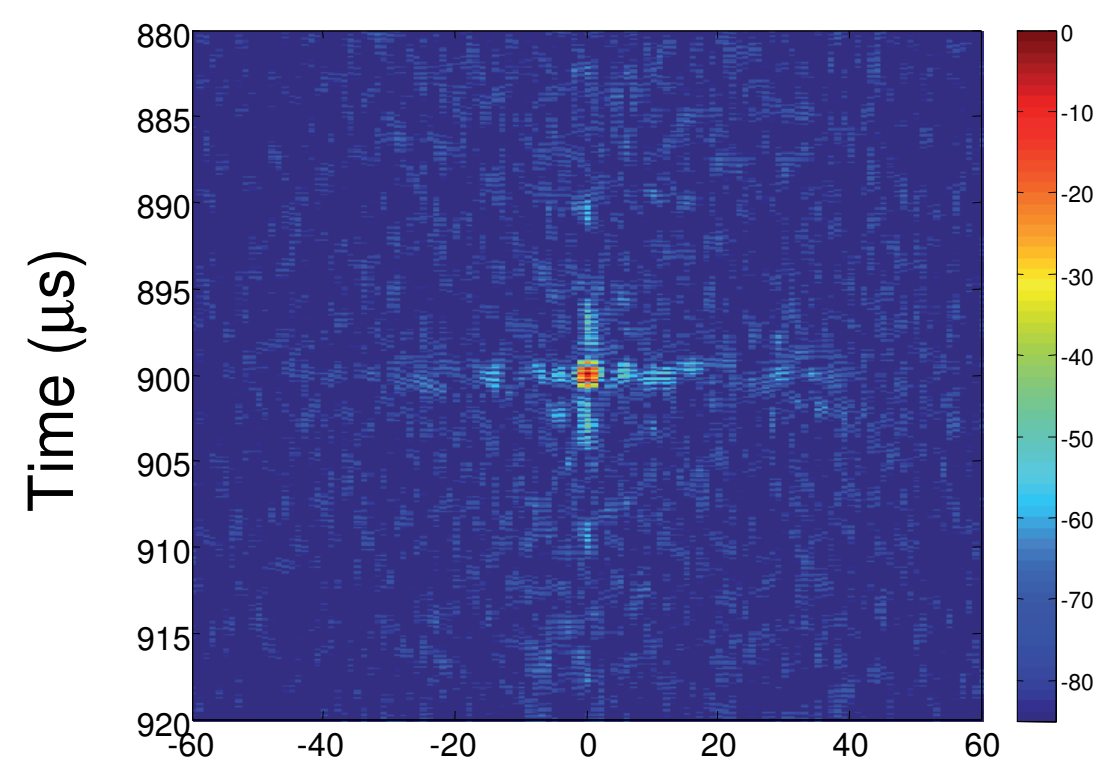

Distance from focal point $(\lambda)$
Wavefront shaping

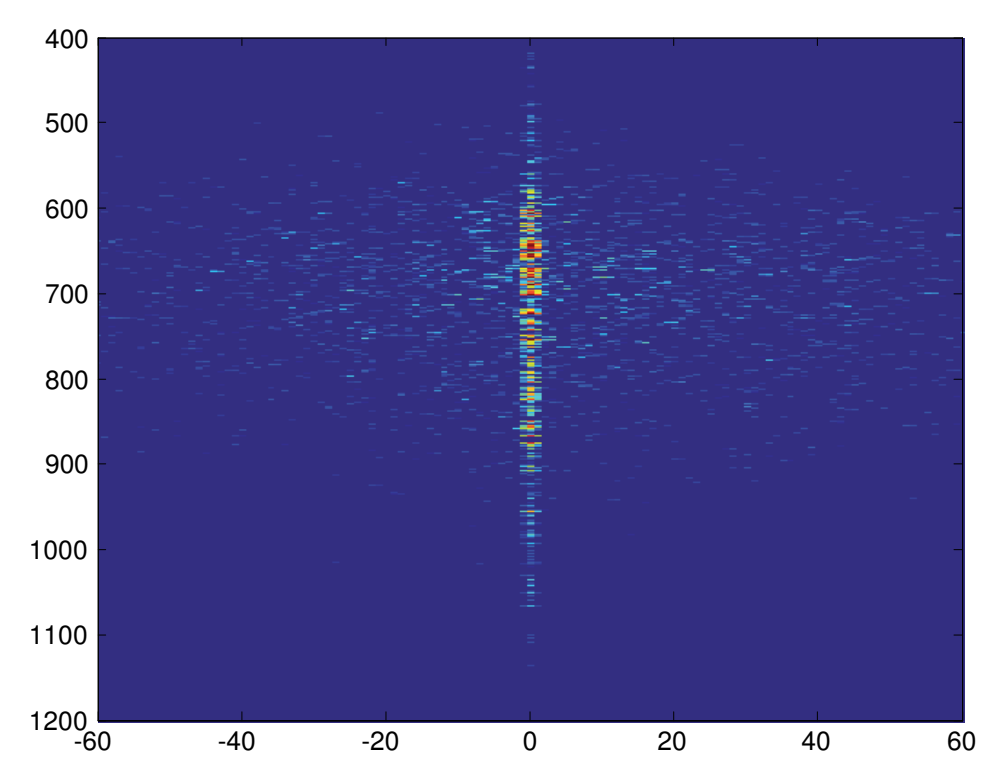

Distance from focal point $(\lambda)$ 


\section{A random lens}

\section{Broadband case}

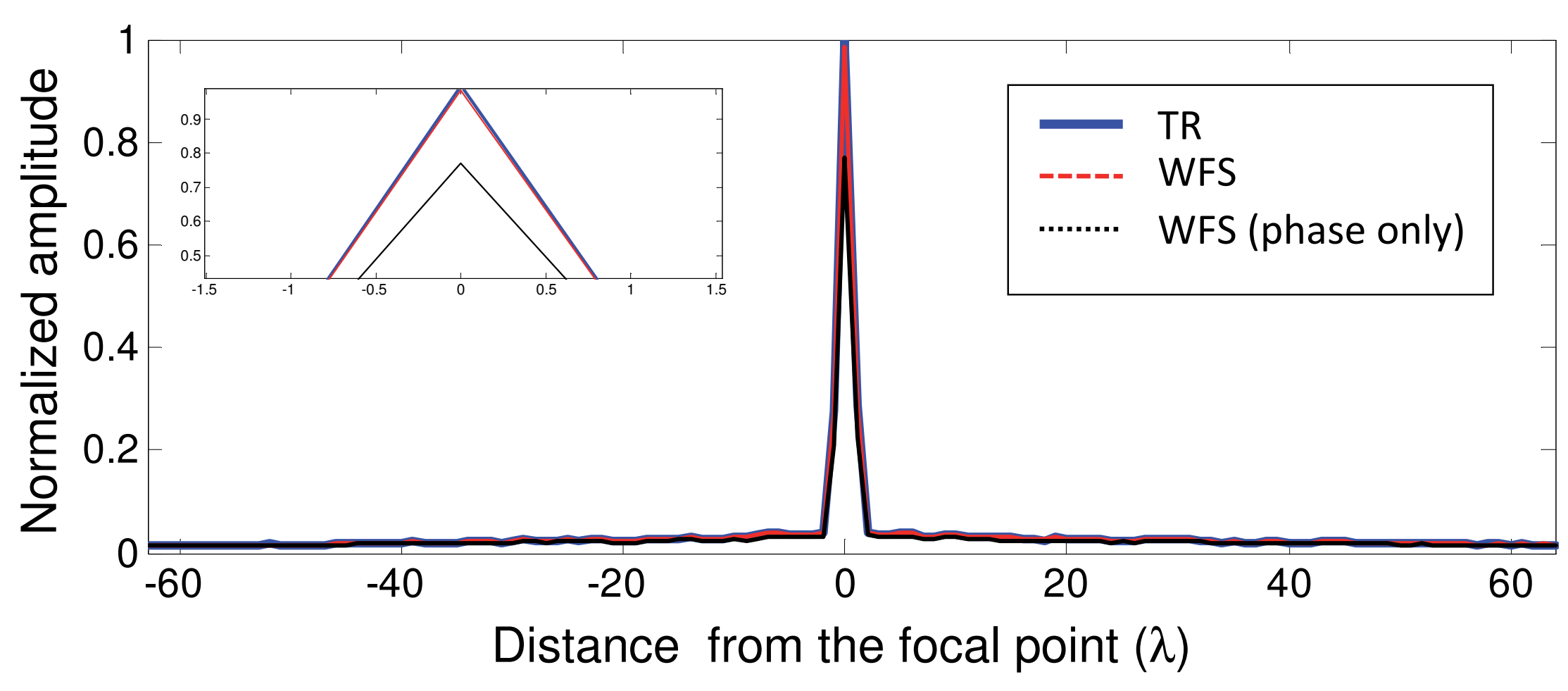




\section{A random (or periodic) bubbly medium}

\section{Minnaert Resonance}
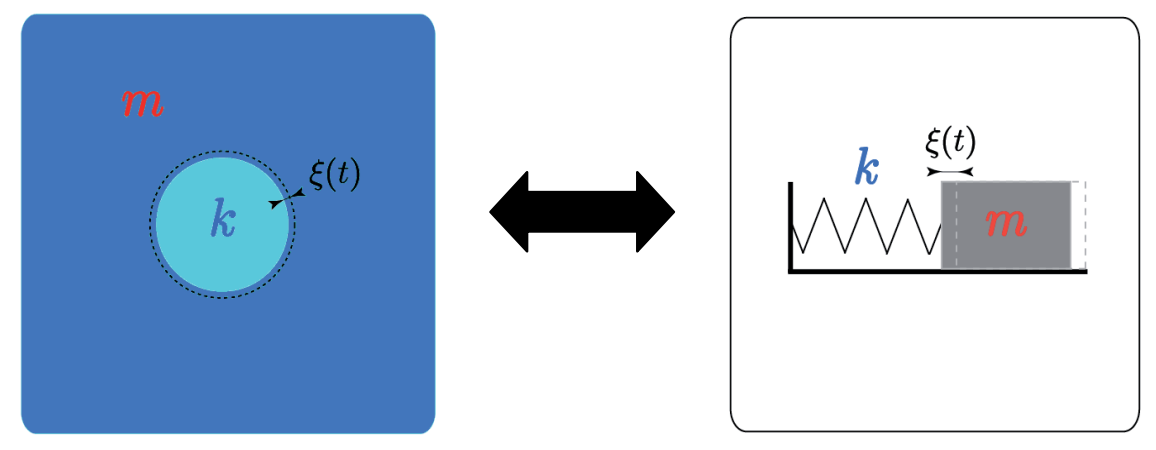

$$
\omega_{M}=\sqrt{\frac{k}{m}}=\frac{1}{R_{0}} \sqrt{\frac{3 \beta_{\text {air }}}{\rho_{\text {eau }}}}
$$

$R=10 \mu \mathrm{m} \quad \longrightarrow \quad f_{M}=300 \mathrm{kHz}$

$$
\sigma_{s}>>\sigma_{g e o}
$$

M. Kafesaki et al.

Air Bubbles in water : a strongly Multiple Scattering Medium for Acoustic Waves

Phys. Rev. Lett. 84, 6050 (2000)

\section{$10 \%$ volume fraction}

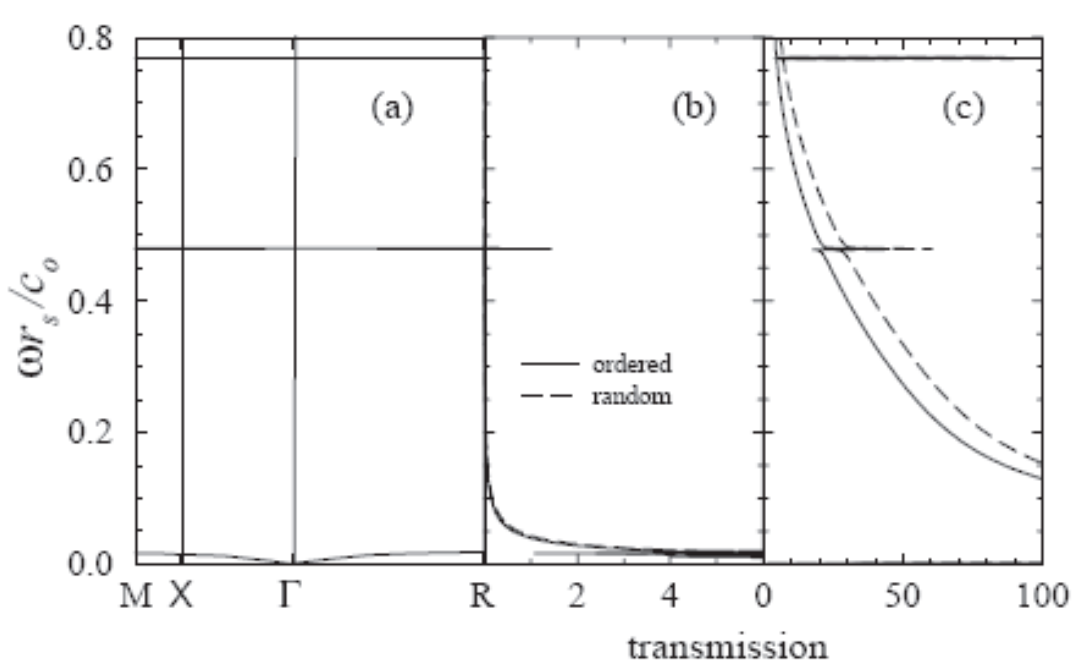




\section{A random (or periodic) bubbly medium}

V. Leroy, A. Bretagne, M. Fink, H. Willaime, P. Tabeling, A. Tourin

"Design and characterization of bubble phononic crystals »

App. Phys. Lett. 95, 171904 (2009)

$h=78 \mu m, a_{x}=200 \mu m, a_{z}=360 \mu m$
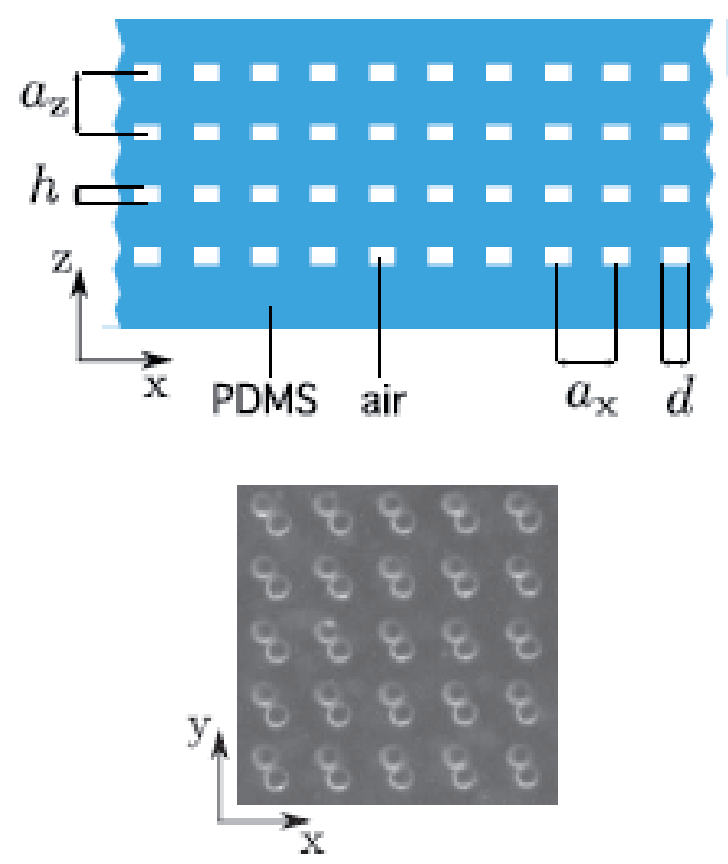

NATURE|Vol 462|24/31 December 2009

\section{APPLIED PHYSICS}

\section{Bubbly but quiet}

\section{Edwin L. Thomas}

An array of air bubbles in a rubber-like material can be made to block the transmission of sound. This finding might help in the design of soundproof walls for music rooms and urban apartments.

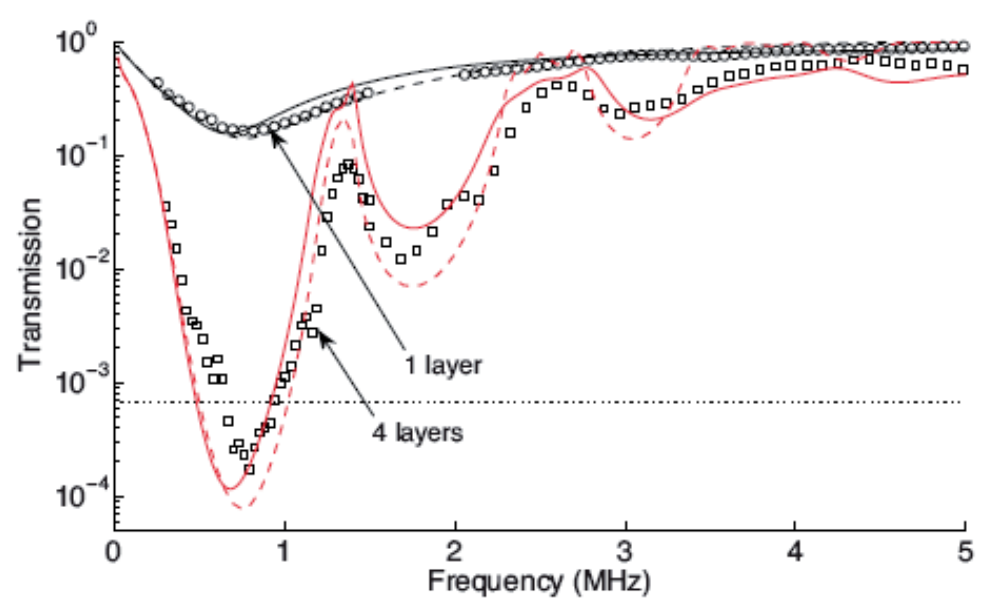


Thank you for your attention! 